Submitted 1/12/93 to the SIAM Journal on Numerical Analysis

\title{
Preconditioning Legendre Spectral Collocation Approximations to Elliptic Problems
}

\author{
by \\ Seymour V. Parter ${ }^{1}$ \\ and \\ Ernest E. Rothman ${ }^{2}$
}

\footnotetext{
1 Departments of Mathematics and Computer Sciences, University of Wisconsin-Madison, Madison, WI 53706. Supported by the NSF under Grant number DMS-9203502

2 Advanced Computing Research Institute, Cornell Center for Theory and Simulation in Science and Engineering. This research was partially supported by the Cornell Theory Center, which receives major funding from the National Science Foundation and IBM Corporation, with additional support from the State of New York and members of its Corporate Research Institute.
} 


\begin{abstract}
This work deals with the $H^{1}$ condition numbers and the distribution of the $\tilde{\beta}_{N, M^{-}}$ singular values of the preconditioned operators $\left\{\tilde{\beta}_{N, M}^{-1} W_{N, M} \hat{A}_{N, M}\right\} . \hat{A}_{N, M}$ is the matrix representation of the Legendre Spectral Collocation discretization of the elliptic operator $A$ defined by $A u:=-\Delta u+a_{1} u_{x}+a_{2} u_{y}+a_{0} u$ in $\Omega$ (the unit square) with boundary conditions: $u=0$ on $\Gamma_{0}, \frac{\partial u}{\partial \nu_{A}}=\alpha u$ on $\Gamma_{1} \cdot \tilde{\beta}_{N, M}$ is the stiffness matrix associated with the finite element discretization of the positive definite elliptic operator $B$ defined by $B v:=-\Delta v+b_{0} v$ in $\Omega$ with boundary conditions $v=0$ on $\Gamma_{0}, \frac{\partial v}{\partial \nu_{B}}=\beta v$ on $\Gamma_{1}$. The finite element space is either the space of continuous functions which are bilinear on the rectangles determined by the Legendre-Gauss-Lobatto (LGL) points or the space of continuous functions which are linear on a triangulation of $\Omega$ determined by the LGL points. $W_{N, M}$ is the matrix of quadrature weights. When $A=B$ we obtain results on the eigenvalues of $\tilde{\beta}_{N, M}^{-1} W_{N, M} \hat{B}_{N, M}$. We show that there is an integer $N_{0}$ and constants $\alpha, \beta$ with $0<\alpha<\beta$, such that: if $\min (N, M) \geq N_{0}$, then all the $\tilde{\beta}_{N, M \text {-singular values }}$ of $\tilde{\beta}_{N, M}^{-1} W_{N, M} \hat{A}_{N, M}$ lie in the interval $[\alpha, \beta]$. Moreover, there is a smaller interval, $\left[\alpha_{0}, \beta_{0}\right]$, independent of the operator $A$, such that: if $\min (N, M) \geq N_{0}$, then all but a fixed finite number of the $\tilde{\beta}_{N, M}$-singular value lie in $\left[\alpha_{0}, \beta_{0}\right]$. These results are related to results of Manteuffel and Parter [MP] Parter and Wong [PW] and Wong [W1], [W2] for finite element discretizations.
\end{abstract}




\section{Introduction} by

Let $\Omega$ be the square $[-1,1] \times[-1,1]$ and consider a uniformly elliptic operator given

$$
A u:=-\left[u_{x x}+u_{y y}\right]+a_{1} u_{x}+a_{2} u_{y}+a_{0} u \text { in } \Omega
$$

with boundary conditions

$$
u=0 \text { on } \Gamma_{0}, \quad \frac{\partial u}{\partial \nu}=\alpha u \text { on } \Gamma_{1}
$$

where

$$
\partial \Omega=\Gamma_{0} \cup \Gamma_{1}
$$

and $\Gamma_{0}\left(\Gamma_{1}\right)$ consists of complete edges of the square, e.g., we could have

$$
\Gamma_{0}=\{(-1, y),-1 \leq y \leq 1\} .
$$

While many of our major results are valid (Theorem 7.1, Theorem 8.3) in the general case of variable coefficients, we limit this discussion to the case where $a_{1}, a_{2}$, and $a_{0}$ are constants. We assume that $A$ is an invertible operator, but not necessarily definite. Let $\left\{A_{N, M}\right\}$ be a family of spectral collocation discretizations based on the Legendre-GaussLobatto $[\mathrm{LGL}]$ points which arise from a variational or weak representation of the operator $A$ (see $[\mathrm{QZ}]$ or $[\mathrm{BM}]$ ). Consider the systems of linear equations.

$$
\hat{A}_{N, M} U=F
$$

which arise in the numerical solution of the boundary value problem

$$
A u=f
$$

using these spectral collocation discretizations and the Lagrange basis $\left\{\varphi_{i j}(x, y)\right\}$ of the polynomial space $P_{N, M}^{0}$ (see Section 2 for a complete discussion of notations, etc).

The actual solution of the system (1.4) is difficult (see [QZ]) because the matrix $\hat{A}_{N, M}$ is badly conditioned. This is true even in the case when $A$ is a symmetric, positive definite operator. Preconditioned iterative methods are a preferred approach (see [CHQZ]). Building on an early suggestion of Orszag $[\mathrm{Or}]$, who used a finite difference preconditioner, several authors have suggested the use of finite element preconditioners ([QZ], [CQ], [DM]). Let $\tilde{\beta}_{N, M}$ be the stiffness matrix of the finite element discretization and let $M_{N, M}$ be the associated mass matrix. A natural approach is to replace (1.4) by

$$
\tilde{\beta}_{N, M}^{-1} M_{N, M} \hat{A}_{N, M} U=\tilde{\beta}_{N, M}^{-1} M_{N, M} F
$$

The solution of (1.5) would then be effected by a damped Jacobi iterative method, GMRES or Bi-CGSTAB [V]. However, these methods can only be effectively used when the eigenvalues of the preconditioned matrix $\beta_{N, M}^{-1} M_{N, M} \hat{A}_{N, M}$ all have positive real parts. Since 
one usually uses a positive definite preconditioner $\tilde{\beta}_{N, M}$ such approaches can be used only when $A$ is itself definite, i.e., its eigenvalues have positive real parts. In the more general case one could consider the Conjugate Gradient method applied to the normal equations associated with (1.5).

Another preconditioning of (1.4) is given by

$$
\tilde{\beta}_{N, M}^{-1} W_{N, M} \hat{A}_{N, M} U=\tilde{\beta}_{N, M}^{-1} W_{N, M} F,
$$

where $W_{N, M}$ is the diagonal matrix of the quadrature weights $\omega_{k} \hat{\omega}_{j}$ associated with the Gauss-Lobatto quadrature. There has been quite a bit of research, mostly experimental, on the eigenvalues of the preconditioned matrices

$$
\tilde{\beta}_{N, M}^{-1} M_{N, M} \hat{A}_{N, M}, \quad \tilde{\beta}_{N, M}^{-1} W_{N, M} \hat{A}_{N, M}
$$

For example, in a recent paper [QZ] the authors describe a modification of (1.4) and carry out a series of interesting computational experiments on the eigenvalues and the solution efficacy of their method. The finite element space employed in [QZ] is the space of continuous piecewise bilinear functions, $V_{N, M}^{0}$, with the basis being the tensor product of the one dimensional "hat" functions.

In this work we consider the same finite element space with the same basis as well as the space of continuous piecewise linear functions, $Z_{N, M}^{0}$, with the basis of two dimensional "hat" functions (see $[\mathrm{J}]$ ). We give a complete analysis of the $\tilde{\beta}_{N, M}$ - singular values of

$$
L_{N, M}:=\tilde{\beta}_{N, M}^{-1} W_{N, M} \hat{A}_{N, M}
$$

the preconditioned matrix associated with (1.6). The matrix $\tilde{\beta}_{N, M}$ is the stiffness matrix of any symmetric positive definite operator of the form

$$
B v=-\left[v_{x x}+v_{y y}\right]+b v \text { in } \Omega
$$

with boundary conditions

$$
u=0 \text { on } \Gamma_{0}, \quad \frac{\partial u}{\partial v}=\beta u \text { on } \Gamma_{1}
$$

While it is essential that the $\Gamma_{0}$ (and $\Gamma_{1}$ ) of $(1.8 \mathrm{~b})$ be the same as the $\Gamma_{0}$ (and $\Gamma_{1}$ ) of (1.1b), there are no other conditions, i.e. we allow

$$
\alpha \neq \beta
$$

Indeed, we will carry out the discussion for the case $\beta=0$. We do this for definiteness and because this is probably as good a choice as any for practical reasons. This flexibility in the choice of boundary conditions is consistent with results of [MP, Theorem 3.2].

The preconditioning results are contained in Theorem 7.1, Theorem 8.3 and Theorem 7.2, Theorem 8.4 which we restate as follows 
Theorem 7.1'. There is an integer $N_{0}(A)$ such that if $\min (N, M) \geq N_{0}$, then the operators $A_{N, M}$ are uniformly invertible. Assume

$$
\min (N, M) \geq N_{0}
$$

There are two positive constants, $0<\alpha<\beta$, independent of $(N, M)$, such that for all $U=\left(u_{1}, u_{2}, \ldots, u_{d}\right)^{T} \neq 0,\left(d=\right.$ dimension $\left.P_{N, M}^{0}\right)$ we have the inequalities

$$
0<\alpha^{2} \leq \frac{\left(\tilde{\beta}_{N, M}\left[L_{N, M} U\right],\left[L_{N, M} U\right]\right)_{l_{2}}}{\left(\tilde{\beta}_{N, M} U, U\right)_{l_{2}}} \leq \beta^{2} .
$$

Theorem 7.2'. Let $\alpha_{0}, \beta_{0}$ be the constants of Theorem $7.1^{\prime}$ associated with the special case where $A=B$. That is, let

$$
Q_{N, M}:=\tilde{\beta}_{N, M}^{-1} W_{N, M} \hat{B}_{N, N} .
$$

From theorem $7.1^{\prime}$ we have, for $U \neq 0$,

$$
0<\alpha_{0}^{2} \leq \frac{\left(\tilde{\beta}_{N, M}\left[Q_{N, M} U\right],\left[Q_{N, M} U\right]\right)_{l_{2}}}{\left(\tilde{\beta}_{N, M} U, U\right)_{l_{2}}} \leq \beta_{0}^{2}
$$

Returning to the general invertible operator $A$; there is an integer $N_{1} \geq N_{0}(A)$ such that if $\min (N, M) \geq N_{1}$ and $\sigma_{j}(N, M)$ are the $\tilde{\beta}_{N, M}$-singular values of $L_{N, M}$, then these singular values cluster in the interval

$$
K:=\left[\left(\frac{\alpha_{0}^{3}}{\beta_{0}}\right)^{1 / 2},\left(\frac{\beta_{0}^{3}}{\alpha_{0}}\right)^{1 / 2}\right] .
$$

The precise statement about clustering is: for $\epsilon>0$ there is an integer $k=k(\epsilon)$ such that all of the $\tilde{\beta}_{N, M}$-singular values, $\sigma_{j}(N, M)$, lie in $K$ - with the exception of $k$ of them.

We observe that the interval $K$ does not depend upon the operator $A$. Theorem 7.1' remains true in the general case of variable coefficients $a_{1}(x, y), a_{2}(x, y), a_{0}(x, y)$. Due to technical details of the weighted discrete inner product used in the formulation of the spectral collocation methods, we can only prove Theorem $7.2^{\prime}$ for the case of constant coefficients. Hence, for simplicity we deal only with case of constant coefficients $a_{1}, a_{2}, a_{0}$.

Since these results are in the $\tilde{\beta}_{N, M}$-norm they imply eigenvalue results as well. In particular, when $A$ is a positive definite self-adjoint operator, $W_{N, M} \hat{A}_{N, M}$ is a symmetric positive definite matrix and the $\tilde{\beta}_{N, M}$-singular values of $L_{N, M}$ are also the eigenvalues of $L_{N, M}$. In general, if $\lambda_{j}(N, M)$ are the eigenvalues of $L_{N, M}$ then

$$
\min \sigma_{j}(N, M) \leq\left|\lambda_{j}(N, M)\right| \leq \max \sigma_{j}(N, M) .
$$

These results enable one to apply the Conjugate Gradient method to the $\tilde{\beta}_{N, M}$ normal equations in the $\tilde{\beta}_{N, M}$-inner product. This approach has been discussed in $[\mathrm{BP}]$ and 
[PW]. The details given there explain and clarify the implementation problems. However, it is also pertinent to mention an interesting experimental fact which will become clear from the computational results of Section 9. The usual singular values, i.e. the square roots of the eigenvalues of $\left(L_{N, M}^{*} L_{N, M}\right)$ seem to have the same distribution properties as the $\tilde{\beta}_{N, M}$-singular values. We have no theoretical explanation of these computational results. However, they imply that - in practice, without proof - one could employ the Conjugate Gradient on the usual normal equations in the usual $\left(\ell_{2}\right)$ inner product.

Our arguments depend upon the theory of preconditioning and boundary conditions developed in $[\mathrm{MP}]$ and extended in [PW], [W1], [W2], [GMP], [G] for finite element equations and some new very powerful estimates on interpolation at the (LGL) points developed in $[\mathrm{M}]$ and $[\mathrm{BM}]$. While the full development of our results is technically complicated, the basic idea is relatively simple. The ideas developed in $[\mathrm{FMP}],[\mathrm{MP}],[\mathrm{PW}]$, and $[\mathrm{W}]$ allow one to develop the general theory provided that one has obtained the basic result of Theorem 7.1 in the special case where

$$
A=B
$$

That is, we are concerned with

$$
Q_{N, M}=\tilde{\beta}_{N, M}^{-1} W \hat{B}_{N, M}
$$

In this case we have the two basic facts

$$
\left(W \hat{B}_{N, M} U, U\right)_{\ell_{2}} \sim\left\|I_{N, M} u\right\|_{1}^{2}
$$

where $u(x, y) \in V_{N, M}^{0}\left(Z_{N, M}^{0}\right)$ and $\left(I_{N, M} u\right) \in P_{N, M}^{0}$ is its polynomial interpolant. Thus, we need only prove the existence of positive constants $0<\alpha^{\prime}<\beta^{\prime}$, independent of (N,M), such that

$$
\alpha^{\prime}\|u\|_{1} \leq\left\|I_{N, M} u\right\|_{1} \leq \beta^{\prime}\|u\|_{1}
$$

The upper bound in (1.13) is provided by the results of $[\mathrm{M}]$ and $[\mathrm{BM}]$. Our task is to establish the lower bound. This is done in Sections 3,4,5 for the case of $V_{N, M}^{0}$ by building on one dimensional results. In Section 8 we establish the existence of positive constants $0<\alpha^{\prime \prime}<\beta^{\prime \prime}$ such that

$$
\alpha^{\prime \prime}\|u\|_{1} \leq\left\|K_{N, M} u\right\|_{1} \leq \beta^{\prime \prime}\|u\|_{1}
$$

where $u \in V_{N, M}^{0}$ and $\left(K_{N, M} u\right) \in Z_{N, M}^{0}$ is its piecewise linear interpolant. The results for $Z_{N, M}^{0}$ then follow from those for $V_{N, M}^{0}$.

In Section 2 we describe the spaces and the notation. In Section 3 we develop some one dimensional estimates relating to polynomials $P_{N}^{0}$ and the continuous piecewise linear 
functions defined by their values at the (LGL) points, $V_{N}^{0}$. Within this narrow context we extend the results of $[\mathrm{M}]$ and $[\mathrm{BM}]$ to show equivalence of both the $L_{2}$ norm and the $H_{1}$ norm of a function and its LGL interpolant. Section 4 is devoted to the study of a basic one-dimensional operators where $A=B$. In section 5 we extend equivalence of the $L_{2}$ and $H_{1}$ norms of polynomials $p(x, y) \in P_{N, M}^{0}$ with their piecewise bilinear interpolants. In Section 6 we deal with preconditioning within $P_{N, M}^{0}$. That is, $\hat{A}_{N, M}$ is multiplied by $\left(\hat{B}_{N, M}\right)^{-1}$ where $\hat{B}_{N, M}$ is the spectral collocation matrix associated with the operator $B$. Section 7 is devoted to the matrix $L_{N, M}$ for the space $V_{N, M}^{0}$. In Section 8 we establish the estimate (1.14) and state the results for $L_{N, M}$ for the space $Z_{N, M}^{0}$. Section 9 describes some computational experiments, both one dimensional and two dimensional.

We are indebted to David Gottlieb for suggesting this research project and our collaboration on it. He also was a patient listener and provided advice and encouragement. We are extremely grateful to Paul Nevai, who gave advice and helpful information and estimates on orthogonal polynomials.

\section{Preliminaries}

In this work we deal with many vector spaces and use relatively standard notations. For example:

a.) if $U=\left(u_{k}\right), V=\left(v_{k}\right)$ are $N$-tuples [or $(N, M)$-tuples ] of real numbers then

$$
(U, V)_{\ell_{2}}:=\sum_{(k)} u_{k} v_{k}
$$

b.) if $u(x), v(x)[$ or $u(x, y), v(x, y)]$ are real functions defined on $[-1,1][$ or $\Omega]$ then

$$
(u, v)_{L_{2}},\|u\|_{L_{2}},\|u\|_{s} \text { denote the }
$$

usual $L_{2}$ inner product, $L_{2}$-norm or $H_{s}$-norm.

There are many occasions when we want to express the fact two families of positive quantities $\left\{a_{N}\right\},\left\{b_{N}\right\}$, [or $\left.\left\{a_{N, M}\right\},\left\{b_{N, M}\right\}\right]$ are uniformly equivalent in the sense that: there are positive constants, $(\alpha, \beta)$, independent of $N$, [ or $(N, M)]$ such that

$$
0<\alpha a_{N}<b_{N}<\beta a_{N}, \quad \forall N .
$$

Rather than repeat this phrase over and over, we write

$$
a_{N} \sim b_{N} .
$$

Definition: Let $T$ be a real positive definite $d \times d$ matrix. The bilinear form

$$
(U, V)_{T}:=(T U, V) \ell_{2}
$$

is an inner product. Let $S$ be any other real $d \times d$ matrix. The $T$-adjoint of $S$ is that unique matrix $S^{\sharp}$ such that

$$
(S U, V)_{T}=\left(U, S^{\sharp} V\right)_{T} \text {. }
$$


It is easy to see that

$$
S^{\sharp}=T^{-1} S^{T} T \text {. }
$$

The $T$-singular values of $S$ are the square roots of the eigenvalues of $S^{\sharp} S$. We denote these singular values by $\sigma_{j}(S: T)$ with

$$
\sigma_{1}(S: T) \geq \sigma_{j}(S: T) \geq \sigma_{j+1}(S: T) \geq \sigma_{d}(S: T)
$$

Finally, the usual min - max characterization of the $\sigma_{j}^{2}(S: T)$ holds. In our case $T=\tilde{\beta}\left(\tilde{\beta}_{N}\right.$ or $\left.\tilde{\beta}_{N, M}\right)$ and $S=\tilde{\beta}^{-1}(W \hat{A})\left(W_{N} \hat{A}_{N}\right.$ or $\left.W_{N, M} \hat{A}_{N, M}\right)$. Hence $S^{\sharp}=$ $\tilde{\beta}^{-1}(W \hat{A})^{*}$ and the $\beta$-singular values are the square roots of the eigenvalues of the matrix.

$$
S^{\sharp} S=\left[\tilde{\beta}^{-1}(W \hat{A})^{*}\right]\left[\tilde{\beta}^{-1}(W \hat{A})\right] .
$$

Let $N$ be a positive integer and let $P_{N}$ denote the set of polynomials of degree $N$ or less. $P_{N}^{0}$ is a subspace of $P_{N}$ which satisfies an additional constraint, that is

$$
P_{N}^{0}:=\left\{f \in P_{N}: f(x)=0 \text { for } x \in \gamma_{0}\right\}
$$

where $\gamma_{0}$ is a subset of $\{-1,1\}$ which may be empty.

Let $N$ and $M$ be positive integers and let $P_{N, M}$ denote the set of all functions of $(x, y)$ which are polynomials in $x$ of degree $N$ or less and are polynomials in $y$ of degree $M$ or less. Set

$$
P_{N, M}^{0}:=\left\{f \in P_{N, M}: f(x, y)=0 \text { for }(x, y) \in \Gamma_{0}\right\} .
$$

Let $\left\{x_{k}\right\}, k=0,1, \cdots, N$; be the Legendre-Gauss-Lobatto (LGL) points associated with the $(N+1)$ point quadrature rule. That is

$$
x_{0}=-1, \quad x_{N}=1
$$

and the intermediate values $-1<x_{1}<x_{2} \cdots<x_{N-1}<1$ are the roots of

$$
\frac{d}{d x} L_{N}(x)=0
$$

where $L_{N}$ is the Legendre polynomial of degree $N$. Let $\left\{\omega_{k}\right\}, k=0,1 \cdots N$ be the associated quadrature weights. Then

$$
\sum_{k=0}^{N} \omega_{k} f\left(x_{k}\right)=\int_{0}^{1} f(x) d x, \quad \forall f \in P_{2 N-1}
$$

where $P_{2 N-1}$ is the set of polynomials of degree $2 N-1$ or less. Similarly, let $\left\{y_{j}\right\}, j=$ $0, \cdots, M$, be the (LGL) points associated with the $(M+1)$ point quadrature rule. Let $\hat{\omega}_{j}$ be the associated quadrature weights. 
In this work we use the Lagrange basis for $P_{N}, P_{N}^{0}, P_{N, M}$ and $P_{N, M}^{0}$. That is, we take

$$
\begin{aligned}
& \varphi_{i}(x)=\left[\left(\prod_{k \neq i}\right)\left(x-x_{k}\right)\right] /\left[\left(\begin{array}{c}
\prod_{k \neq i} \\
k \neq
\end{array}\right)\left(x_{i}-x_{k}\right)\right] \\
& \hat{\varphi}_{j}(x)=\left[\left(\begin{array}{c}
\prod_{j \neq s} \\
j \neq y
\end{array}\left(y-y_{s}\right)\right] /\left[\left(\begin{array}{c}
\prod_{j \neq s} \\
)
\end{array}\left(y_{j}-y_{s}\right)\right]\right.\right.
\end{aligned}
$$

where, of course, the $x_{k}$ and $y_{s}$ are the (LGL) points. Then the set $\left\{\varphi_{i}(x) ; i=\right.$ $0,1, \cdots, N\}$ is the basis for $P_{N}$. The same set, with some possible deletions, is the basis for $P_{N}^{0}$. Similarly, the set $\left\{\varphi_{i j}(x, y)=\varphi_{i}(x) \hat{\varphi}_{j}(y) ; \quad i=0,1, \cdots, N ; \quad j=0,1, \cdots, M\right\}$ is the basis for $P_{N, M}$. And, the same set - with the necessary deletions - is the basis for $P_{N, M}^{0}$. We define the discrete inner products

$$
\begin{gathered}
\langle f, g\rangle_{N}:=\sum_{(k)} \omega_{k} f\left(x_{k}\right) g\left(x_{k}\right), \quad \forall f, g \in P_{N}, \\
\langle f, g\rangle_{N, M}:=\sum_{k} \sum_{j} \omega_{k} \hat{\omega}_{j} f\left(x_{k}, y_{j}\right) g\left(x_{k}, y_{j}\right), \quad \forall f, g \in P_{N, M}
\end{gathered}
$$

The discrete norms are given by

$$
\begin{gathered}
\|f\|_{N}:=\left[\langle f, f\rangle_{N}\right]^{1 / 2}, \quad \forall f \in P_{N} \\
\|f\|_{N, M}:=\left[\langle f, f\rangle_{N, M}\right]^{1 / 2}, \quad \forall f \in P_{N, M}
\end{gathered}
$$

We also require a discrete boundary inner-product and a discrete boundary integral. In order to express this inner-product in complete detail we introduce the following definitions and notations. We number the sides of $\Omega$

$$
\begin{array}{ll}
s_{1}= & \text { Side } 1:=\{(x,-1):-1 \leq x \leq 1\} \\
s_{2}= & \text { Side } 2:=\{(1, y):-1 \leq y \leq 1\} \\
s_{3}= & \text { Side } 3:=\{(x, 1):-1 \leq x \leq 1\} \\
s_{4}= & \text { Side } 4:=\{(-1, y):-1 \leq y \leq 1\}
\end{array}
$$

We define a boundary inner-product for each side. Thus

$$
[f, g]_{N, M, 1}:=\sum_{k=0}^{N} f\left(x_{k},-1\right) g\left(x_{k},-1\right) \omega_{k}
$$




$$
[f, g]_{N, M, 2}:=\sum_{j=0}^{M} f\left(1, y_{j}\right) g\left(1, y_{j}\right) \hat{\omega}_{j}
$$

$$
[f, g]_{N, M, 3}:=\sum_{k=0}^{N} f\left(x_{k}, 1\right) g\left(x_{k}, 1\right) \omega_{k}
$$

$$
[f, g]_{M, M, 4}:=\sum_{j=0}^{M} f\left(-1, y_{j}\right) g\left(-1, y_{j}\right) \hat{\omega}_{j}
$$

If $\Gamma_{1}=$ union of sides $\mu_{s}$ then

$$
[f, g]_{N, M, \Gamma_{1}}=\sum_{(s)}[f, g]_{N, M, \mu_{s}}
$$

The boundary integral is defined by

$$
\|f\|_{N, M, \Gamma_{1}}=\left([f, f]_{N, M, \Gamma_{1}}\right)^{1 / 2}
$$

The space $V_{N}\left(V_{M}\right)$ consists of the continuous piecewise linear functions defined on $[-1,1]$ which are defined by their values at the points $x_{k}\left(y_{j}\right)$. That is, $u \in V_{N}$ if $u \in C[-1,1]$ and $u$ is linear on each interval $\left(x_{k}, x_{k+1}\right), k=1,2, \cdots, N$. The space $V_{N}^{0}\left(V_{M}^{0}\right)$ is the subspace of $V_{N}\left(V_{M}\right)$ consisting of functions which vanish on $\gamma_{0}$. The basis $\left\{\psi_{i}(x), i=0,1, \cdots, N\right\}$ of $V_{N}$ is given by the usual "hat" functions. These functions satisfy

$$
\psi_{i}\left(x_{k}\right)=\delta_{i k}
$$

The basis of $V_{N}^{0}$ is the same set with the necessary deletions. The basis for $V_{M}$ and $V_{M}^{0}$ is given by $\left\{\hat{\psi}_{j}(y) ; j=0,1, \cdots, M\right\}$, the corresponding hat functions based on the $(M+1)$ LGL points, with the appropriate deletions in the case of $V_{M}^{0}$.

Consider the partition of $\Omega$ into rectangles $K$ whose vertices are LGL points $\left(x_{k}, y_{j}\right),\left(x_{k+1}, y_{j}\right),\left(x_{k+1}, y_{j+1}\right),\left(x_{k}, y_{j+1}\right)$. The space $V_{N, M}$ is the set of continuous functions $u$ which are bilinear, (of the form $u=a+b x+c y+d x y$ ) on each such rectangle $K$. The basis of this space is the set $\left\{\psi_{i j}(x, y)=\psi_{i}(x) \hat{\psi}_{j}(y): \quad 0 \leq i \leq N, 0 \leq j \leq M\right\}$. the space $V_{N, M}^{0}$ is the subspace of $V_{N, M}$ which vanishes on $\Gamma_{0}$. The basis for $V_{N, M}^{0}$ is the basis for $V_{N, M}$ with appropriate deletions

Let $K$ be the rectangle above. Let $K$ be partitioned into two triangles $T_{1}, T_{2}$ by drawing the diagonal connecting $\left(x_{k+1}, y_{j}\right)$ and $\left(x_{k}, y_{j+1}\right)$. The space $Z_{N, M}$ is the space of continuous functions $w$ which are linear on each such triangle. The basis for $Z_{N, M}$ is the interpolatory basis based on two dimensional "hat" functions. That is, as in $V_{N, M}$, the "degrees of freedom" are the values at the LGL points. The space $Z_{N, M}^{0}$ is the subspace of $Z_{N, M}$ which vanishes on $\Gamma_{0}$. The basis for $Z_{N, M}^{0}$ is the basis for $Z_{N, M}$ with appropriate deletions. 
Definition. Let $u \in V_{N}$ and

$$
u(x)=\sum_{k=0}^{N} u_{k} \psi_{k}(x)
$$

Then $\left(I_{N} u\right) \in P_{N}$, the polynomial interpolant of $u$, is given by

$$
\left(I_{N} u\right)(x)=\sum_{k=0}^{N} u_{k} \varphi_{k}(x) \in P_{N}
$$

Clearly, this map is 1 to 1 and onto. The inverse map is denoted by $J_{N}$. That is, if $f \in P_{N}$ and

$$
f(x)=\sum f_{k} \varphi_{k}(x)
$$

then

$$
\left(J_{N} f\right)(x)=\sum f_{k} \psi_{k}(x) \in V_{N}
$$

In a similar fashion we define $I_{N, M}$ and $J_{N, M}$. That is, if

$$
u(x, y)=\sum u_{k j} \psi_{k j}(x, y) \in V_{N, M}
$$

then

$$
\left(I_{N, M} u\right)(x, y)=\sum u_{k j} \varphi_{k j}(x, y) \in P_{N, M} .
$$

And, if

$$
f(x, y)=\sum f_{k j} \varphi_{k j}(x, y) \in P_{N, M}
$$

then

$$
\left(J_{N, M} f\right)(x, y)=\sum f_{k j} \psi_{k j}(x, y) \in V_{N, M} .
$$

The interpolation operator $K_{N, M}$ takes functions $u \in V_{N, M}^{0}$ into functions $w \in Z_{N, M}^{0}$ which agree at the LGL points. Specifically, if $K$ is the rectangle described above with vertices $\left(x_{k}, y_{j}\right),\left(x_{k+1}, y_{j}\right),\left(x_{k+1}, y_{j+1}\right),\left(x_{k}, y_{j+1}\right)$ and $u(x, y) \in V_{N, M}^{0}$ is given on $K$ by

$$
\left.u\right|_{K}=a+b\left(x-x_{k}\right)+c\left(y-y_{j}\right)+d\left(x-x_{k}\right)\left(y-y_{j}\right)
$$

Then

$$
\left.K_{N, M} u\right|_{K}=\left.w\right|_{K}= \begin{cases}a+b\left(x-x_{k}\right)+c\left(y-y_{j}\right) & \text { on } T_{1} \\ \alpha+\beta\left(x-x_{k+1}\right)+\gamma\left(y-y_{j+1}\right) & \text { on } T_{2}\end{cases}
$$


where $T_{1}$ is the triangle with vertices $\left(x_{k}, y_{j}\right),\left(x_{k+1}, y_{j}\right),\left(x_{k}, y_{j+1}\right)$ and $T_{2}$ is the triangle with vertices $\left(x_{k+1}, y_{j}\right),\left(x_{k+1}, y_{j+1}\right),\left(x_{k}, y_{j+1}\right)$ and

$$
\begin{gathered}
\alpha=a+b\left(x_{k+1}-x_{k}\right)+c\left(y_{j+1}-y_{j}\right)+d\left(x_{k+1}-x_{k}\right)\left(y_{j+1}-y_{j}\right) \\
\beta=b+d\left(y_{j+1}-y_{j}\right) \\
\gamma=c+d\left(x_{k+1}-x_{k}\right)
\end{gathered}
$$

A consequence of the fact that our bases are interpolatory at the same points $\left(x_{k}\right)$ or $\left(x_{k}, y_{j}\right)$, is that we can interpret coefficient vectors as representing $u \in V_{N}$ or $I_{N} u \in$ $P_{N}$, etc. Thus, if we have (2.16a) we may interpret the $U=\left(u_{0}, u_{1}, \cdots, u_{N}\right)^{T}$ as the representor of $u(x) \in V_{N}$. Or, if we so desire, we may also interpret $U$ as the representor of $\left(I_{N} u\right)(x) \in P_{N}$. Similar remarks apply to the two dimensional vectors $\left(u_{1}, u_{2}, \cdots, u_{d}\right)^{T}$ which may be interpretted as representing $u(x, y) \in V_{N, M}^{0},\left(I_{N, M} u\right) \in P_{N, M}^{0}$ or $\left(K_{N, M} u\right) \in$ $Z_{N, M}^{0}$.

If $f, g \in P_{N}$ and

$$
F=\left(f_{o}, f_{1}, \cdots, f_{N}\right)^{T}, \quad G=\left(g_{0}, g_{1}, \cdots, g_{N}\right)^{T}
$$

are the vectors of coefficients then

$$
\langle f, g\rangle_{N}=\left(W_{N} F, G\right)_{\ell_{2}}
$$

where

$$
W_{N}=\operatorname{diagonal}\left(w_{0}, w_{1}, \cdots, w_{N}\right)
$$

Similarly if $f, g \in P_{N, M}$ and

$$
F=\left\{f\left(x_{i}, y_{j}\right)\right\}, \quad G=\left\{g\left(x_{i}, y_{j}\right)\right\}
$$

are the "vectors" of coefficients then

$$
\langle f, g\rangle_{N, M}=\left(W_{N, M} F, G\right)_{\ell_{2}}
$$

where

$$
W_{N, M}=\text { diagonal }\left(\omega_{k} \hat{\omega}_{j}\right)
$$

A more precise description of $W_{N, M}$ will be given later.

There are many spaces, $P_{N}^{0}, P_{N, M}^{0}, V_{N}^{0}$, etc. and these are operators and matrices etc. We adopt the following notational conventions: 
(1.) Operators mapping $P_{N}^{0} \rightarrow P_{N}^{0}$ or $P_{N, M}^{0} \rightarrow P_{N, M}^{0}$ are denoted by capital Roman letters with subscripts. For example

$$
B_{N}: P_{N}^{0} \rightarrow P_{N}^{0}, \quad A_{N, M}: P_{N, M}^{0} \rightarrow P_{N, M}^{0}
$$

(2.) The matrix representations of these operators in the Lagrange basis are denoted by the same capital Roman letters with subscripts and a "hat". For example, the matrix representation of $B_{N}$ is $\hat{B}_{N}$.

(3.) Operators mapping $V_{N}^{0} \rightarrow V_{N}^{0}$ or $V_{N, M}^{0} \rightarrow V_{N, M}^{0}$ are denoted by lower case Greek letters with subscripts. For example

$$
\beta_{N}: V_{N}^{0} \rightarrow V_{N}^{0}, \quad \beta_{N, M}: V_{N, M}^{0} \rightarrow V_{N, M}^{0}
$$

(4.) The matrix representations of these operators in the $\left\{\psi_{i}(x)\right\}$ or $\left\{\psi_{i j}(x, y)\right\}$ basis are denoted by the same lower case Greek letters with subscripts and a "hat". For example, the matrix representation of $\beta_{N}$ is $\hat{\beta}_{N}$.

(5.) When $\beta_{N}$ or $\left(\beta_{N, M}\right)$ is the mapping associated with finite element discretization of a differential operator, the stiffness matrix associated with $\beta_{N}$ or $\left(\beta_{N, M}\right)$ in the Lagrange basis is denoted by the same lower case Greek letter with subscripts and a "tilde". That is, $\tilde{\beta}_{N}$ or $\tilde{\beta}_{N, M}$.

In Section 8 we consider the finite element discretization of $B$ in the space $Z_{N, M}^{0}$. For that Section only, $\tilde{\beta}_{N, M}$ denotes the stiffness matrix of that finite element discretization.

\section{One Dimensional Estimates}

In this section we collect some basic estimates relating $u \in V_{N}$ and $\left(I_{N} u\right) \in P_{N}$. We first recall results of $[\mathrm{CQ}],[\mathrm{M}]$ and $[\mathrm{BM}]$.

Theorem CQ. For every $f \in P_{N}$ we have

$$
\|f\|_{L_{2}}^{2} \sim<f, f>_{N}
$$

For every $f \in P_{N, M}$ we have

$$
\|f\|_{L_{2}}^{2} \sim<f, f>_{N, M}
$$

Proof. $\quad$ See [CQ. page 83].

Theorem M. There is a constant $C$, independent of $N$ such that

$$
\left\|I_{N} u\right\|_{1} \leq C\|u\|_{1}, \quad \forall u \in V_{N}
$$

Proof. The results of $[\mathrm{M}]$ are more general. However, this is the result we require in the sequel. 
Lemma 3.1. Let $\omega_{k}$ and $x_{k}$ be the $L G L$ weights and the $L G L$ points respectively. Then

$$
\begin{gathered}
\omega_{k} \sim\left(x_{k+1}-x_{k-1}\right), \quad k=1,2, \cdots, N-1, \\
\omega_{0} \sim\left(x_{1}-x_{0}\right)=1+x_{1} \\
w_{N} \sim\left(x_{N}-x_{N-1}\right)=1-x_{N-1} .
\end{gathered}
$$

Proof. $\quad$ For $k=0$ and $N$ we have

$$
\omega_{0}=w_{N}=\frac{2}{N(N+1)} .
$$

Thus the estimates (3.4b), follow immediately from the estimates on the distribution of the zeros of $L_{N}(x)$ given in [S, theorem 6.21.3].

The Legendre polynomials satisfy the differential equation

$$
\frac{d}{d x}\left(1-x^{2}\right) \frac{d}{d x} L_{N}=N(N+1) L_{N} .
$$

Hence, the functions $L_{N}^{\prime}(x)$ are the orthogonal polynomials of degree $(N-1)$ associated with the weight function $\left(1-x^{2}\right)$. We use the notation of $[\mathrm{N}]$ and denote the quadrature weights (or Cote's numbers) of the Gauss quadrature with weight $w=\left(1-x^{2}\right)$ by $\lambda_{N-1}\left(w, \xi_{j, N+1}\right)$. Of course $\xi_{j, N-1}=x_{j}, \quad j=1,2, \cdots,(N-1)$. It is immediate that

$$
\omega_{j}=\frac{\lambda_{N-1}\left(w, \xi_{j, N-1}\right)}{1-\left(\xi_{j, N-1}\right)^{2}} .
$$

Using $[\mathrm{N}$, Theorem 6.3 .28 , page 120$]$ we see that

$$
\omega_{j} \sim \frac{\sqrt{1-x_{j}^{2}}}{N}, \quad j=1,2, \cdots, N
$$

However, it follows from [N, Theorem 9.22, page 166] that

$$
\frac{\sqrt{1-x_{j}^{2}}}{N} \sim\left(x_{j+1}-x_{j-1}\right) .
$$

Thus, the Lemma is proven.

Theorem 3.1. For all $u \in V_{N}$ we have

$$
\|u\|_{L_{2}} \sim\left\|I_{N} u\right\|_{L_{2}}
$$




$$
\|u\|_{L_{2}} \sim\left\|I_{N} u\right\|_{N}
$$

Proof. The statements (3.7a) and (3.7b) are equivalent by (3.1) of Theorem CQ. We shall prove $(3.7 \mathrm{~b})$. A direct computation shows that

$$
\int_{x_{j}}^{x_{j+1}} u^{2}(t) d t=\frac{x_{j+1}-x_{j}}{6}\left[u\left(x_{j}\right)^{2}+u\left(x_{j}\right) u\left(x_{j+1}\right)+u\left(x_{j+1}\right)^{2}\right] .
$$

Let

$$
T:=\left[\left(1+x_{1}\right) u(-1)^{2}+\left(1-x_{N-1}\right) u(1)^{2}+\sum_{k=1}^{N-1}\left(x_{k+1}-x_{k-1}\right) u\left(x_{k}\right)^{2}\right] .
$$

Then (3.8) shows that

$$
\frac{1}{6} T \leq\|u\|_{L_{2}}^{2} \leq \frac{1}{3} T .
$$

The theorem now follows from Lemma 3.1.

Using this result we can strengthen Theorem M.

Theorem 3.2. For all $u \in V_{N}$ we have

$$
\|u\|_{1} \sim\left\|I_{N} u\right\|_{1}
$$

Proof. In view of Theorem M and Theorem 3.1 we need only prove

$$
\int_{-1}^{1}\left|u^{\prime}\right|^{2} d t \leq \int_{-1}^{1}\left|\left(I_{N} u\right)^{\prime}\right|^{2} d t
$$

Observe that

$$
\int_{x_{k}}^{x_{k}+1}\left|u^{\prime}(t)\right|^{2} d t=\frac{\left[u\left(x_{k+1}\right)-u\left(x_{k}\right)\right]^{2}}{x_{k+1}-x_{k}}
$$

and that

$$
\left[u\left(x_{k+1}\right)-u\left(x_{k}\right)\right]^{2}=\left[\int_{x_{k}}^{x_{k}+1}\left(I_{N} u\right)^{\prime} d t\right]^{2} .
$$

The Schwarz inequality yields

$$
\left[u\left(x_{x+1}\right)-u\left(x_{k}\right)\right]^{2} \leq\left[\int_{x_{k}}^{x_{k+1}}\left[\left(I_{N} u\right)^{\prime}\right]^{2} d t\right]\left(x_{k+1}-x_{k}\right) .
$$

Thus (3.9) follows from (a) dividing (3.11c) by $\left(x_{k+1}-x_{k}\right)$ and (b) summing on $k$. 


\section{The Basic 1D Preconditioning}

We consider two positive definite self-adjoint differential operators $B^{1}, B^{2}$ defined on $C_{2}[-1,1]$. Then we describe the spectral collocation discretization of $B^{1}$ in $P_{N}^{0}$ and the finite element discretization of $B^{2}$ in $V_{N}^{0}$. Finally, we discuss the preconditioned matrix.

$$
Q:=\left(\tilde{\beta}_{N}^{2}\right)^{-1} W_{N} \hat{B}_{N}^{1}
$$

where $\tilde{\beta}_{N}^{2}$ is the "stiffness" matrix associated with the finite element discretization of $B^{2}$ and $\hat{B}_{N}^{1}$ is the matrix representation of the spectral collocation discretization of $B^{1}$.

Actually, it would be sufficient for our purposes to consider the case of one operator $B$ and deal with the preconditioned matrix

$$
Q_{1}:=\left(\tilde{\beta}_{N}\right)^{-1} W_{N} \hat{B}_{N}
$$

where $\tilde{\beta}_{N}$ is the associated stiffness matrix and $\hat{B}_{N}$ is the matrix representation of the spectral collocation of $B$. However we consider the more general case to illustrate the basic ideas and emphasize the fact it is not necessary that these operators have the same boundary conditions. Indeed, it is necessary and sufficient only that the points at which one imposes the "essential" boundary condition, $u=0$, be the same for both operators.

Consider the differential operators given by

$$
B^{k} u:=-u^{\prime \prime}+b_{k, 0} u, \quad-1<x<1, k=1,2,
$$

with boundary conditions

$$
u=0 \text { on } \gamma_{0}, \quad u^{\prime}=d_{k} u \text { on } \gamma_{1}
$$

where

$$
\gamma_{0} \cup \gamma_{1}=\{-1,1\} .
$$

The coefficients $b_{k, 0}$ are non-negative constants and

$$
d_{k}(-1) \geq 0, \quad d_{k}(1) \leq 0 .
$$

Both operators are to be positive definite. Hence, if $\gamma_{0}=\phi$ then either $b_{k, 0}>0$ or $\left|d_{k}(-1)\right|+\left|d_{k}(1)\right|>0$.

For simplicity and definiteness we will assume $\gamma_{0}=\{-1\}, \quad \gamma_{1}=\{1\}$. The reader will observe that the argument is completely general.

Associated with these operators are bilinear forms $b_{k}(\cdot, \cdot)$, which are the basis of both the finite element method and the variational form of the spectral collocation approximation of the operators $B^{1} ; B^{2}$. Let

$$
V:=\left\{u \in H_{1}[-1,1], u(-1)=0\right\}
$$

Then $b_{k}: V \times V \rightarrow R, k=1,2$, are defined by

$$
b_{k}(u, v):=\int_{-1}^{1}\left[u^{\prime} v^{\prime}+b_{k, 0} u v\right] d x-d_{k}(1) u(1) v(1) .
$$

Each of these bilinear forms is an inner product on $V$ and the associated norms given by

$$
\|u\|_{1, k}=\left[b_{k}(u, u)\right]^{1 / 2}, \quad k=1,2 .
$$

are equivalent to the $H_{1}$ norm on $V$. Thus, we have 
Lemma 4.1. For all $u \in V$ we have

$$
\begin{aligned}
\|u\|_{1,1}^{2}=b_{1}(u, u) & \sim b_{2}(u, u)=\|u\|_{1,2}^{2}, \\
b_{1}(u, u) & \sim\|u\|_{1}^{2} \cdot \square
\end{aligned}
$$

We set

$$
\begin{aligned}
& P_{N}^{0}:=\left\{f \in P_{N}: f(-1)=0\right\}, \\
& V_{N}^{0}:=\left\{u \in V_{N}: u(-1)=0\right\} .
\end{aligned}
$$

Let $b_{1, N}(\cdot, \cdot)$ be defined on $P_{N}^{0} \times P_{N}^{0}$ by

$$
b_{1, N}(f, g):=\left\langle f^{\prime}, g^{\prime}\right\rangle_{N}+b_{1,0}\langle f, g\rangle_{N}-d_{1}(1) f(1) g(1),
$$

and let $b_{2, N}(\cdot, \cdot)$ be defined on $V_{N}^{0} \times V_{N}^{0}$ by

$$
b_{2, N}(u, v):=b_{2}(u, v), \quad \forall u, v \in V_{N}^{0} .
$$

These bilinear forms induce operators $B_{N}^{1}, \beta_{N}^{2}$ which are given by

$$
\begin{gathered}
B_{N}^{1}: P_{N}^{0} \rightarrow P_{N}^{0}, \quad \beta_{N}^{2}: V_{N}^{0} \rightarrow V_{N}^{0}, \\
\left\langle B_{N}^{1} f, g\right\rangle_{N}=b_{1, N}(f, g), \quad \forall f, g \in P_{N}^{0} . \\
\left(\beta_{N}^{2} u, v\right)_{L_{2}}=b_{2, N}(u, v), \quad \forall u, v \in V_{N}^{0} .
\end{gathered}
$$

Let $\left\{\varphi_{k}(x) ; k=1,2, \cdots, N\right\}$ be the Lagrange basis of $P_{N}^{0}$. Let $\hat{B}_{N}^{1}$ be the matrix representation of $B_{N}^{1}$ in this basis. Then (see [QZ])

$$
\left(\hat{B}_{N}^{1}\right)_{i j}=\left(B^{1} \varphi_{j}\right)\left(x_{i}\right)+\frac{1}{\omega_{N}}\left[\varphi_{j}^{\prime}\left(x_{N}\right)-d_{1}(1) \varphi_{j}\left(x_{i}\right)\right] \delta_{i, N}
$$

Let $\left\{\psi_{k}(x) ; k=1,2, \cdots, N\right\}$ be the basis of $V_{N}^{0}$. Let $\tilde{\beta}_{N}^{2}$ be the stiffness matrix associated with the finite element treatment of this problem and let $M_{N}$ be the mass matrix. That is

$$
\begin{gathered}
\left(\tilde{\beta}_{N}^{2}\right)_{i j}=b_{2, N}\left(\psi_{i}, \psi_{j}\right) . \\
\left(M_{N}\right)_{i j}=\left(\psi_{i}, \psi_{j}\right)_{L_{2}} .
\end{gathered}
$$

Let $\hat{\beta}_{N}^{2}$ be the matrix representation of $\beta_{N}^{2}$. Then

$$
\hat{\beta}_{N}^{2}=M_{N}^{-1} \tilde{\beta}_{N}^{2} \text {. }
$$

Lemma 4.2. For all $u \in V_{N}^{0}$ and all $f \in P_{N}^{0}$ we have

$$
\begin{aligned}
& b_{1, N}(f, f) \sim\|f\|_{1}^{2} \\
& b_{2, N}(u, u) \sim\|u\|_{1}^{2}
\end{aligned}
$$

Proof. The estimates (4.13b) follow directly from Lemma 4.1. The estimates (4.13a) follow from Theorem $\mathrm{CQ}$ and the fact that

$$
\left\langle f^{\prime}, g^{\prime}\right\rangle_{N}=\int_{-1}^{1} f^{\prime} g^{\prime} d x .
$$

The operator $B_{N}^{1}$ is symmetric and positive definite in the $\langle\cdot, \cdot\rangle$ inner product. Hence the matrix

$$
\tilde{B}_{N}^{1}=W_{N} \hat{B}_{N}^{1}
$$

is symmetric and positive definite in the $\ell_{2}$ inner product. Thus we have 
Lemma 4.3. Let

$$
\begin{aligned}
& f(x)=\sum f_{j} \varphi_{j}(x) \in P_{N}^{0} \\
& u(x)=\sum u_{j} \psi_{j}(x) \in V_{N}^{0}
\end{aligned}
$$

and let

$$
F=\left(f_{1}, f_{2}, \cdots, f_{N}\right)^{T}, \quad U=\left(u_{1}, u_{2}, \cdots, u_{N}\right)^{T}
$$

be the coefficient vectors. Then

$$
\begin{gathered}
\left(W_{N} \hat{B}_{N}^{1} F, F\right)_{\ell_{2}}=b_{1, N}(f, f) \sim\|f\|_{1}^{2} \\
\left(\tilde{\beta}_{N}^{2} U, U\right)_{\ell_{2}}=b_{2}(u, u) \sim\|u\|_{1}^{2}
\end{gathered}
$$

Proof. Immediate from the definitions.

Theorem 4.1. For every $U=\left(u_{1}, \cdots, u_{N}\right)^{T}$ we have

$$
\left(\tilde{\beta}_{N}^{2} U, U\right)_{\ell_{2}} \sim\left(W_{N} \hat{B}_{N}^{1} U, U\right)_{\ell_{2}}
$$

In matrix theory language, there are constants $0<\alpha_{0}<\beta_{0}$ independent of $N$ such that the eigenvalues $q_{j}$ of the matrix

$$
Q=\left(\tilde{\beta}_{N}^{2}\right)^{-1} W \hat{B}_{N}^{1}
$$

satisfy

$$
\alpha_{0} \leq q_{j} \leq \beta_{0}
$$

Proof. Let $u(x) \in V_{N}^{0}$ be defined by

$$
u(x)=\sum_{j=1}^{N} u_{j} \psi_{j}(x) .
$$

Then

$$
\left(I_{N} u\right)(x)=\sum_{j=1}^{N} u_{j} \phi_{j}(x) .
$$

Thus, the vector $U$ represents $u(x)$ and also represents $\left(I_{N} u\right)$. That is

$$
\left(\tilde{\beta}_{N}^{2} U, U\right)_{\ell_{2}}=b_{2}(u, u) \sim\|u\|_{1}^{2}
$$

and

$$
\left(W_{N} \hat{B}_{N}^{1} U, U\right)_{\ell_{2}}=b_{1, N}\left(I_{N} u, I_{N} u\right) \sim\left\|I_{N} u\right\|_{1}^{2}
$$

The theorem now follows from Theorem 3.2. 


\section{The Basic 2D Preconditioning}

Consider the elliptic differential operator

$$
B v:=-\left[v_{x x}+v_{y y}\right]+v, \quad(x, y) \in \Omega,
$$

with boundary conditions

$$
v=0 \text { on } \Gamma_{0}, \quad \frac{\partial u}{\partial \gamma}=0 \text { on } \Gamma_{1} .
$$

In this section we describe both the spectral collocation approximation to $B$ in $P_{N, M}^{0}$ and the finite element approximation to $B$ in $V_{N, M}^{0}$. Our concern is the preconditoned matrix.

$$
Q_{N, M}:=\left(\tilde{\beta}_{N, M}\right)^{-1} W_{N, M} \hat{B}_{N, M}
$$

where $\tilde{\beta}_{N, M}$ is the stiffness matrix of the finite element method and $\hat{B}_{N, M}$ is the matrix representation of the spectral collocation discretization of $B$. Let

$$
V:=\left\{u \in H_{1}(\Omega), \quad u=0 \text { on } \Gamma_{0}\right\}
$$

where the boundary condition on $\Gamma_{0}$ is taken in the sense of the trace theorem. Associated with the operator $B$ is the bilinear form $b(\cdot, \cdot)$ defined on $V \times V$ given by

$$
b(u, v)=(\nabla u, \nabla v)_{L_{2}}+(u, v)_{L_{2}}, \quad \forall u, v \in V .
$$

It is well known that

$$
b(u, u) \sim\|u\|_{1}^{2} .
$$

Let

$$
\begin{gathered}
P_{N, M}^{0}:=\left\{f \in P_{N, M}: f=0 \text { on } \Gamma_{0}\right\} \\
V_{N, M}^{0}:=\left\{u \in V_{N, M}: u=0 \text { on } \Gamma_{0}\right\} .
\end{gathered}
$$

Let $b_{N, M}(\cdot, \cdot)$ be defined on $P_{N, M}^{0} \times P_{N, M}^{0}$ by

$$
b_{N, M}(f, g)=\langle\nabla f, \nabla g\rangle_{N, M}+\langle f, g\rangle_{N, M}, \quad \forall f, g \in P_{N, M}^{0} .
$$

This bilinear form defines the operator

$$
B_{N, M}: P_{N, M}^{0} \rightarrow P_{N, M}^{0}
$$

via the equation

$$
b_{N, M}(f, g)=\left\langle B_{N, M} f, g\right\rangle_{N, M}, \quad \forall f, g \in P_{N, M}^{0} .
$$

This operator $B_{N, M}$ is the spectral collocation discretization of the operator $B$. 
Lemma 5.1. For every $f \in V_{N, M}^{0}$ we have

$$
\|f\|_{1}^{2} \sim b_{N, M}(f, f) .
$$

Proof. We have

$$
b_{N, M}(f, f)=\langle\nabla f, \nabla f\rangle_{N, M}+\langle f, f\rangle_{N, M} .
$$

The basic result of [CQ: page 83$]$ shows that

$$
\|f\|_{L_{2}}^{2} \sim\langle f, f\rangle_{N, M}
$$

Hence we need only deal with the first term. Consider the expression $\left\langle f_{x}, f_{x}\right\rangle_{N, M}$. We have

$$
\left\langle f_{x}, f_{x}\right\rangle_{N, M}=\sum_{j=0}^{M} \tilde{\omega}_{j} \int_{-1}^{1} f_{x}^{2}\left(t, y_{j}\right) d t
$$

Consider the function

$$
g(y):=\int_{-1}^{1} f_{x}^{2}(t, y) d t
$$

This function is a polynomial of even order of degree $2 M$ or less which is non-negative for $-\infty<y<\infty$. Therefore by [S, Theorem 1.21 .2 page 5]

$$
g(y)=A(y)^{2}+B(y)^{2}
$$

where $A(y)^{2} \in P_{2 M}, B(y)^{2} \in P_{2 M}$. Thus by Theorem CQ we have

$$
\left\langle f_{x}, f_{x}\right\rangle_{N, M} \sim \int_{-1}^{1} g(y) d y=\int_{-1}^{1} \int_{-1}^{1} f_{x}^{2}(t, y) d t d y .
$$

Thus, the Lemma is proven.

The finite element discretization, $\beta_{N, M}$, of the operator $B$ is defined by

$$
\beta_{N, M}: V_{N, M}^{0} \rightarrow V_{N, M}^{0}
$$

and

$$
b(u, v)=\left(\beta_{N, M} u, v\right)_{L_{2}}, \quad \forall u, v \in V_{N, M}^{0} .
$$

Lemma 5.2. For every $u \in V_{N, M}^{0}$ we have

$$
b(u, u)=\left(\beta_{N, M} u, u\right)_{L_{2}} \sim\|u\|_{1}^{2}
$$


Proof. The Lemma follows immediately from (5.4).

We now turn to the matrix representations of these operators and their properties. Let us order the LGL points by horizontal lines. For example, if $\Gamma_{1}=\partial \Omega$ so that all the LGL points appear in our computation and we list the LGL points as $P_{1}, P_{2}, \cdots, P_{(N+1)(M+1)}$ then

$$
\left(x_{k}, y_{j}\right)=P_{\mu}
$$

where

$$
\mu=(k+1)+(N+1) j
$$

We order the basis vectors $\varphi_{i j}(x, y) \in P_{N, M}^{0}, \psi_{i j}(x, y) \in V_{N, M}^{0}$ with $k=0,1, \cdots, N$ and $j=0,1, \cdots, M$, in the same order. We define

$$
\begin{aligned}
& \Psi_{\mu}(x, y)=\psi_{k j}(x, y)=\psi_{k}(x) \tilde{\psi}_{j}(y), \\
& \Phi_{\mu}(x, y)=\varphi_{k j}(x, y)=\varphi_{k}(x) \tilde{\varphi}_{j}(y) .
\end{aligned}
$$

Because of the multiplicative structure of these basis functions it is easy to see that these matrix representations have a tensor product structure. For example, let $\tilde{\beta}_{N, M}$ be the stiffness matrix of the finite element discretization and let $M_{N, M}$ be the mass matrix.

Then

$$
\left(\tilde{\beta}_{N, M}\right)_{\mu, \nu}=b\left(\Psi_{\mu}, \Psi_{\nu}\right)
$$

Given $\mu$ one can compute $k$ and $j$ from (5.15b) by observing that $0 \leq k \leq N$ and $0 \leq j \leq$ $M$. Hence

$$
\begin{aligned}
& k+1=\mu \bmod (N+1), \\
& j=\max \left\{\frac{\mu-(k+1)}{N+1}, 0\right\} .
\end{aligned}
$$

Let $B^{x}$ and $B^{y}$ be the ordinary differential operators

$$
B^{x} u=-u^{\prime \prime}+\frac{1}{2} u, \quad-1 \leq x \leq 1
$$

with boundary conditions

$$
u=0 \text { on } \gamma^{0}(x), \quad u^{\prime}=0 \text { on } \gamma^{1}(x)
$$

and

$$
B^{y} u=-u^{\prime \prime}+\frac{1}{2} u, \quad-1 \leq y \leq 1
$$

with boundary conditions

$$
u=0 \text { on } \gamma_{0}(y), \quad u^{\prime}=0 \text { on } \gamma^{1}(y)
$$

Here

$$
\gamma^{0}(x) \cup \gamma^{1}(x)=\{-1,1\}, \quad \gamma^{0}(y) \cup \gamma^{1}(y)=\{-1,1\}
$$

and these decompositions are related to $\Gamma_{0}$ and $\Gamma_{1}$. For example, if the side $\left(s_{4}\right) \subset \Gamma_{0}$ then $(-1) \in \Gamma^{0}(x)$, etc. 
Theorem 5.1. Let $\tilde{\beta}_{N}^{x}$ be the stiffness matrix associated with the finite element discretization of $B^{x}$ in the finite element space $V_{N}^{0}$. Let $\tilde{\beta}_{M}^{y}$ be the stiffness matrix associated with the finite element discretization of $B^{y}$ in the finite element space $V_{M}^{0}$. Let $M_{N}$ and $M_{M}$ be the corresponding mass matrices. Then

$$
\tilde{\beta}=M_{M} \otimes \tilde{\beta}_{N}^{x}+\tilde{\beta}_{M}^{y} \otimes M_{N}
$$

Proof. Computation.

Theorem 5.2. Let $\hat{B}_{N}^{x}$ be the matrix representation of the spectral collocation discretization of $B^{x}$ in $P_{N}^{0}$ [as discussed in section 4 - e.g., equation 4.10 ] and let $\hat{B}_{M}^{y}$ be the matrix representation of the spectral colocation discretization of $B^{y}$ in $P_{M}^{0}$. Then $\hat{B}_{N, M}$, the matrix representation of the spectral collocation discretization of the operator $B$ in $P_{N, M}^{0}$ is given by

$$
\hat{B}_{N, M}=(i d)_{M} \otimes \hat{B}_{N}^{x}+\hat{B}_{M}^{y} \otimes(i d)_{N}
$$

where $(i d)_{M}$ and $(i d)_{N}$ are the identity matrices. Let

$$
\begin{gathered}
\tilde{B}_{N}^{x}=W_{N} \hat{B}_{N}^{x}, \quad \tilde{B}_{M}^{y}=W_{M} \hat{B}_{M}^{y} \\
\tilde{B}_{N, M}=W_{N, M} \hat{B}_{N, M} .
\end{gathered}
$$

Then

$$
\tilde{B}_{N, M}=W_{M} \otimes \tilde{B}_{N}^{x}+\tilde{B}_{M}^{y} \otimes W_{N}
$$

Proof. Computation.

Remark: As stated in the Introduction, the matrix $\hat{B}_{N, M}$ is not the matrix used in [QZ]. These matrices differ in the rows relating to points of $\Gamma_{1}$

Remark: The matrix $\tilde{B}_{N, M}$ is the symmetric matrix of the spectral collocation equations - see [QZ], the matrices $\tilde{B}_{N}^{x}, \tilde{B}_{M}^{y}$ are symmetric - see section 4 . Hence $\tilde{B}_{N, M}$ is symmetric.

Lemma 5.3. Let

$$
\begin{aligned}
& f(x, y)=\sum f_{\mu} \Phi_{\mu}(x, y) \in P_{N, M}^{0}, \\
& u(x, y)=\sum u_{\mu} \Psi_{\mu}(x, y) \in V_{N, M}^{0} .
\end{aligned}
$$

Let

$$
F=\left(f_{1}, f_{2}, \cdots, f_{(N+1)(M+1)}\right)^{T}
$$




$$
U=\left(u_{1}, u_{2}, \cdots, u_{(N+1)(M+1)}\right)^{T} .
$$

(The number of $f_{\mu}$, and $u_{\mu}$ will in general be less than $(N+1)(M+1)$.) Then

$$
\left(\tilde{\beta}_{N, M} U, U\right)_{\ell_{2}}=b(u, u) \sim\|u\|_{1}^{2}
$$

and

$$
\left(\tilde{B}_{N, M} F, F\right)_{\ell_{2}}=b_{N, M}(f, f) \sim\|f\|_{1}^{2}
$$

Proof. The equality of (5.24a) is a well known fact of finite element theory and follows from (5.12b). The equivalence statement in (5.24a) follows from (5.13). The equality of (5.24b) follows from (5.7b) and (2.25b). The equivalence statement of (5.24b) follows from (5.8).

Lemma 5.4. For every vector $U=\left(u_{1}, \cdots, u_{(N+1)(M+1)}\right)^{T}$ we have

$$
\left(\left(W_{M} \otimes \tilde{B}_{N}^{x}\right) U, U\right)_{\ell_{2}} \sim\left(\left(M_{M} \otimes \tilde{\beta}_{N}^{x}\right) U, U\right)_{\ell_{2}}
$$

and

$$
\left(\left(\tilde{B}_{M}^{y} \otimes W_{N}\right) U, U\right)_{\ell_{2}} \sim\left(\left(\tilde{\beta}_{M}^{y} \otimes M_{N}\right) U, U\right)_{\ell^{2}}
$$

Proof. $\quad$ For every

$$
U^{N}=\left(u_{0}, u_{1}, \cdots, u_{N}\right)^{T}
$$

and

$$
V_{M}=\left(v_{0}, v_{1}, \cdots, v_{M}\right)^{T}
$$

Theorem 3.1 implies that

$$
\begin{gathered}
\left(W_{N} U^{N}, U^{N}\right)_{\ell_{2}} \sim\left(M_{N} U^{N}, U^{N}\right)_{\ell_{2} .}, \\
\left(W_{M} V^{M}, V^{M}\right)_{\ell_{2}} \sim\left(M_{M} V^{M}, V^{M}\right)_{\ell_{2}} .
\end{gathered}
$$

Theorem 4.1 asserts that

$$
\left(\tilde{B}_{M}^{x} U^{N}, U^{N}\right) \ell_{2} \sim\left(\tilde{\beta}_{N}^{x} U^{N}, U^{N}\right) \ell_{2},
$$

and

$$
\left(\tilde{B}_{M}^{y} V^{M}, V^{M}\right)_{\ell_{2}} \sim\left(\tilde{\beta}_{M}^{y} V_{M}, V_{M}\right) \ell_{2} .
$$

Since all the matrices involved are symmetric and positive definite these equivalence statements represent bounds on certain eigenvalues. 
Consider the eigenvalue problems

$$
\begin{aligned}
& W_{N} U^{N}=\mu M_{N} U^{N}, \\
& \tilde{B}_{M}^{y} V^{M}=\lambda \tilde{\beta}_{M}^{y} V^{M} .
\end{aligned}
$$

Each has a complete set of eigenvectors $U^{M}(s), s=1,2, \cdots,(N+1), \quad V^{M}(t), t=$ $1,2, \cdots,(M+1)$. Therefore, the vectors and eigenvalues

$$
Z_{s t}=V^{M}(t) \otimes U^{N}(s), \quad \lambda_{t} \mu_{s}=\Lambda_{s t}
$$

are a complete set of eigenvectors and eigenvalues of the eigenvalue problem

$$
\left(\tilde{B}_{M}^{y} \otimes W_{N}\right) U=\Lambda\left(\tilde{\beta}_{M}^{y} \otimes M_{N}\right) U .
$$

The equivalences $(5.26 \mathrm{a})$ and $(5.27 \mathrm{~b})$ yield the precise statement: There are constants $C_{1}, C_{2}, D_{1}, D_{2}$ all positive and independent of $\mathrm{N}, \mathrm{M}$ such that

$$
\begin{aligned}
& 0<C_{1} \leq \lambda_{t} \leq C_{2}, \\
& 0<D_{1} \leq \mu_{s} \leq D_{2} .
\end{aligned}
$$

Thus,

$$
C_{1} D_{1} \leq \Lambda_{s t} \leq C_{2} D_{2}
$$

and (5.25b) follows. The same argument yields (5.25a).

Theorem 5.3. For every $U=\left(u_{1}, u_{2}, \cdots, u_{(N+1)(M+1)}\right)^{T}$ we have

$$
\left(\tilde{\beta}_{N, M} U, U\right) \ell_{2} \sim\left(\tilde{B}_{N, M} U, U\right) \ell_{2}
$$

This statement is equivalent to: Let $0<q_{1} \leq q_{2} \leq \cdots \leq q_{(N+1)(M+1)}$ be the eigenvalues of the matrix $Q_{N, M}$ given by (5.2). Then there are constants $0<\alpha_{0}<\beta_{0}$ and

$$
0<\alpha_{0} \leq q_{j} \leq \beta_{0}
$$

Proof. The theorem follows immediately from Lemma 5.4.

The same argument also yields.

Theorem 5.4. For every $U=\left(u_{1}, u_{2}, \cdots, u_{(N+1)(M+1)}\right)^{T}$ we have

$$
\left(W_{N, M} U, U\right)_{\ell_{2}} \sim\left(M_{N, M} U, U\right)_{\ell_{2}} .
$$

Theorems 5.3 and 5.4 can be interpreted as extensions of Theorems 3.1 and 3.2. 
Theorem 5.5. For all $u \in V_{N, M}^{0}$ we have

$$
\begin{gathered}
\|u\|_{1} \sim\left\|I_{N, M} u\right\|_{1} \\
\|u\|_{L_{2}} \sim\left\|I_{N, M} u\right\|_{L_{2}}
\end{gathered}
$$

Proof. Let

$$
\begin{gathered}
u(x, y)=\sum u_{\mu} \Psi_{\mu}(x, y) \in V_{N, M}^{0} \\
\left(I_{N, M} u\right)(x, y)=\sum u_{\mu} \Phi_{\mu}(x, y) \in P_{N, M}^{0} .
\end{gathered}
$$

The vector $U=\left(u_{1}, u_{2}, \cdots, u_{(N+1)(M+1)}\right)^{T}$ is the coefficient vector of both functions. The general result of CQ yields

$$
\left\|I_{N, M} u\right\|_{L_{2}}^{2} \sim\left(W_{N, M} U, U\right)_{\ell_{2}}
$$

Standard finite element theory gives

$$
\|u\|_{L_{2}}^{2}=\left(M_{N, M} U, U\right)_{\ell_{2}}
$$

Thus (5.30b) follows from Theorem 5.4. Similarly, (5.30a) follows from (5.29a) and Lemma 5.3.

\section{Preconditioning Within $\mathbf{P}_{\mathrm{N}, \mathrm{M}}^{0}$}

The spectral collocation discretization, $A_{N, M}$, of the operator $A$ given by (1.1) is defined by the bilinear form

$$
\begin{aligned}
a_{N, M}(f, g) & =\langle\nabla f, \nabla g\rangle_{N, M}+a_{1}\left\langle f_{x}, g\right\rangle_{N, M} \\
& +a_{2}\langle f, g\rangle_{N, M}+a_{0}\langle f, g\rangle_{N, M}-\alpha[f, g]_{N, M, \Gamma_{1}}
\end{aligned}
$$

As in (5.7b) we have

$$
a_{N, M}(f, g)=\left\langle A_{N, M} f, g\right\rangle_{N, M} \quad \forall f, g \in P_{N, M}^{0}
$$

In this section we are concerned with the operator

$$
S_{N, M}:=B_{N, M}^{-1} A_{N, M}: P_{N, M}^{0} \rightarrow P_{N, M}^{0}
$$

Our results are essentially the results of [MP:Theorem 3.2] and [PW:Theorem 14]. 
Lemma 6.1. There is a positive integer $N_{0}>0$ and a constant $K_{1}(A)>0$ such that if

$$
\min (N, M) \geq N_{0}
$$

then $A_{N, M}^{-1}$ exists and

$$
\left\|A_{N, M}^{-1}\right\|_{N, M} \leq K_{1}(A)
$$

Proof. This result is immediate from the fact that the eigenvalues and eigenfunctions of $A_{N, M}$ converge to those of $A$ as $\min (N, M) \rightarrow \infty$. A direct proof is easily obtained from the arguments of $[\mathrm{P} 1]$.

Throughout the rest of this paper we assume

$$
\min (N, M) \geq N_{0}
$$

Using Lemma 5.1 and the arguments of [MP, Lemma 3.5, Theorem 3.27], which apply word for word, we obtain

Theorem 6.1. Assume (6.5). For every $f \in P_{N, M}^{0}$ let

$$
g=B_{N, M}^{-1} A_{N, M} f=S_{N, M} f
$$

Then

$$
\begin{gathered}
\left\langle B_{N, M} g, g\right\rangle_{N, M} \sim\left\langle B_{N, M} f, f\right\rangle_{N, M} \\
\left\|S_{N, M} f\right\|_{1} \sim\|f\|_{1} \square
\end{gathered}
$$

Translating this result into a statement about matrices and using Theorem 5.3 we obtain

Theorem 6.2. For every $U=\left(u, \cdots, u_{(N+1)(M+1)}\right)^{T}$ let

$$
Z=\hat{B}_{N, M}^{-1} \hat{A}_{N, M} U
$$

Then

$$
\begin{gathered}
\left(\tilde{B}_{N, M} Z, Z\right)_{\ell_{2}} \sim\left(\tilde{B}_{N, M} U, U\right)_{\ell_{2}} \\
\left(\tilde{\beta}_{N, M} Z, Z\right)_{\ell_{2}} \sim\left(\tilde{\beta}_{N, M} U, U\right)_{\ell_{2}} .
\end{gathered}
$$

The theorems above are essentially the restatement of the results of $[\mathrm{MP}]$ in this context. We now turn to the clustering results of $[\mathrm{PW}]$ and $[\mathrm{G}]$. 
Lemma 6.2. Let

$$
S=B^{-1} A: V \rightarrow V
$$

Then $\left\{S_{N, M}\right\}$ is consistent with $S$. That is for every $f \in V$ and every sequence $f_{N, M} \in$ $P_{N, M}^{0}$ such that

$$
\left\|f_{N, M}-f\right\|_{1} \rightarrow 0
$$

we have

$$
\left\|S_{N, M} f_{N, M} \rightarrow S f\right\|_{1} \rightarrow 0
$$

Proof. $\quad S$ is a bounded operator, see $[\mathrm{MP}]$. The family of operators $\left\{S_{N, M}\right\}$ is uniformly bounded. Hence, it is sufficient to prove (6.9b) for $f$ in a dense set of $V$ and $\left\{f_{N, M}\right\}$ a particular sequence which satisfies $(6.9 \mathrm{a})$. Let $f \in C^{4}(\bar{\Omega})$. Let

$$
g=A f
$$

and let $f_{N, M}$ be the "elliptic interpolant", i.e.

$$
A_{N, M} f_{N, M}=g=A f . \quad \text { (at the collocation points) }
$$

Let

$$
S_{N, M} f_{N, M}=B_{N, M}^{-1} A_{N, M} f_{N, M}=u_{N, M},
$$

and

$$
v=B^{-1} A f=B^{-1} g=S f .
$$

Then

$$
B v=A f=g .
$$

Since the convergence theory of spectral collocation shows that

$$
\left\|u_{N, M}-v\right\|_{1} \rightarrow 0
$$

we see that $(6.9 \mathrm{~b})$ holds.

Observe that since $B_{N, M}$ is self-adjoint in the $\langle\cdot, \cdot\rangle_{N, M}$ inner product we have

$$
\left\langle B_{N, M}^{-1} A_{N, M} f, g\right\rangle_{N, M}=\left\langle A_{N, M} f, B_{N, M}^{-1} g\right\rangle_{N, M}
$$

That is

$$
\left\langle B_{N, M}^{-1} A_{N, M} f, g\right\rangle_{N, M}=a_{N, M}(f, q)
$$


where

$$
q:=B_{N, M}^{-1} g
$$

Let

$$
\left\{\begin{aligned}
\rho_{N, M}(f, q) & =-a_{1}\left\langle f, q_{x}\right\rangle_{N, M}-a_{2}\left\langle f, q_{y}\right\rangle_{N, M} \\
& +\left(a_{0}-1\right)\langle f, q\rangle_{N, M}-\alpha[f, q]_{N, M, \Gamma_{1}} \\
& +a_{1}\left\{[f, q]_{N, M, 2}-[f, q]_{N, M, 4}\right\}+a_{2}\left\{[f, q]_{N, M, 3}-[f, q]_{N, M, 1}\right\}
\end{aligned}\right.
$$

Lemma 6.3. Let $T_{N, M}: P_{N, M}^{0} \rightarrow P_{N, M}^{0}$ be defined by

$$
S_{N, M}=(i d)+T_{N, M}
$$

where (id) denotes the identity operator in $P_{N, M}^{0}$. Let

$$
T=(i d)-S
$$

$T$ is a bounded operator taking $V \rightarrow V$. Then $T_{N, M}$ is consistent with $T$. Moreover $T_{N, M}$ is determined by the bilinear form

$$
\tau_{N, M}(f, g)=\left\langle T_{N, M} f, g\right\rangle_{N, M}=\rho_{N, M}\left(f,\left[B_{N, M}^{-1} g\right]\right) .
$$

Proof. We have

$$
\begin{aligned}
a_{N, M}(f, q) & =b_{N, M}(f, q)+a_{1}\left\langle f_{x}, q\right\rangle_{N, M}+a_{2}\left\langle f_{y}, q\right\rangle_{N, M} \\
& +\left(a_{0}-1\right)\langle f, q\rangle_{N, M}-\alpha[f, q]_{N, M, \Gamma_{1}}
\end{aligned}
$$

Using (6.12a) we see that

$$
\begin{aligned}
\left\langle S_{N, M} f, g\right\rangle_{N, M} & =b_{N, M}(f, q)+a_{1}\left\langle f_{x}, q\right\rangle_{N, M}+a_{2}\left\langle f_{y}, q\right\rangle_{N, M} \\
& +\left(a_{0}-1\right)\langle f, q\rangle_{N, M}-\alpha[f, q]_{N, M, \Gamma_{1}}
\end{aligned}
$$

with

$$
q=B_{N, M}^{-1} g
$$

Note: $b_{N, M}(\cdot, \cdot)$ is defined in (5.6). Since

$$
b_{N, M}\left(f, B_{N, M}^{-1} g\right)=\left\langle B_{N, M} f, B_{N, M}^{-1} g\right\rangle_{N, M}
$$

we see that

$$
\begin{aligned}
\left\langle T_{N, M} f, g\right\rangle_{N, M} & =a_{1}\left\langle f_{x}, q\right\rangle_{N, M}+a_{2}\left\langle f_{y}, q\right\rangle_{N, M} \\
& +\left(a_{0}-1\right)\langle f, q\rangle_{N, M}-\alpha[f, q]_{N, M, \Gamma_{1}} .
\end{aligned}
$$

The final form $(6.14 \mathrm{c})$ comes from integration by parts. 
Lemma 6.4. Let $\min (N, M) \geq N_{0}$. There is a constant $K_{T}$, depending only on $T$, such that

$$
\left\|T_{N, M} f\right\|_{1} \leq K_{T}\|f\|_{3 / 4}
$$

Proof. Let

$$
T_{N, M} f=v .
$$

Because of $(6.14 \mathrm{a})$ and $(6.6 \mathrm{c})$ we see that there is a constant $c_{0}>0$ such that

$$
\|f\|_{1} \leq c_{0}\|v\|_{1} .
$$

Also we see that

$$
B_{N, M} v=A_{N, M} f-B_{N, M} f .
$$

Hence

$$
b_{N, M}(v, g)=\rho_{N, M}(f, g), \forall g \in P_{N, M}^{0} .
$$

In particular

$$
b_{N, M}(v, v)=\rho_{N, M}(f, v) .
$$

Then, using the form of $\rho_{N, M}$ and the equivalence of norms discussed earlier, we see that there is a constant $c_{1}>0$ such that

$$
\|v\|_{1}^{2}=\|\nabla v\|_{L_{2}}^{2}+\|v\|_{L_{2}}^{2} \leq c_{1}\left[\|f\|_{L_{2}}\|v\|_{1}+\left[\int_{\Gamma_{1}}|f|^{2} d \sigma\right]^{1 / 2}\left[\int_{\Gamma_{1}}|v|^{2} d \sigma\right]^{1 / 2}\right]
$$

Consider the boundary integrals. It is well-known that there is a constant $c_{2}$, depending only on $\Omega$, such that

$$
\begin{gathered}
\int_{\Gamma_{1}}|v|^{2} d \sigma \leq c_{2}\|v\|_{1}^{2} \\
\int_{\Gamma_{1}}|f|^{2} d \sigma \leq c_{2}\|f\|_{3 / 4}^{2}
\end{gathered}
$$

Since

$$
\|f\|_{L_{2}} \leq\|f\|_{3 / 4}
$$

We have

$$
\|v\|_{1}^{2} \leq c_{1}\|f\|_{3 / 4}\|v\|_{1}+c_{1} c_{2}\|f\|_{3 / 4}\|v\|_{1} .
$$


We recall the inequality

$$
|a b| \leq 1 / 2\left[\varepsilon|a|^{2}+\frac{1}{\varepsilon}|b|^{2}\right] .
$$

Applying the inequality to (6.22) we find that there is a constant $c_{3}$, depending only on $c_{1}$ and $c_{2}$, such that

$$
\|v\|_{1}^{2} \leq 1 / 2\|v\|_{1}^{2}+c_{3}\|f\|_{3 / 4}^{2}
$$

or

$$
\|v\|_{1}^{2} \leq 2 c_{3}\|f\|_{3 / 4}^{2}
$$

which proves the Lemma.

The results of Lemma 6.2 and Lemma 6.4 enable one to prove the collective compactness [A] of the family $\left\{T_{N, M}\right\}$. The argument which follows is to be found in [W2 : appendix]. The operator $T=S-(i d)$ is a bounded compact operator taking $V \rightarrow V$.

Lemma 6.5. The operators $\left\{T_{N, M}: \min (N, M) \geq N_{0}\right\}$ are collectively compact. That is, let $f_{N, M} \in P_{N, M}^{0}$ and

$$
\left\|f_{N, M}\right\|_{1} \leq C
$$

Then, there is a subsequence $\left(N_{k}, M_{k}\right)$ and a function $g \in V$ such that

$$
\left\|T_{N_{k}, M_{k}} f_{N_{k}, M_{k}}-g\right\|_{1} \rightarrow 0
$$

Proof. Let $\prod_{N, M}$ denote the $H_{1}$ projection onto $P_{N, M}^{0}$ based on $b(\cdot, \cdot)$. That is, for every $u \in V$, we have

$$
\prod_{N, M} u \in P_{N, M}^{0}
$$

and

$$
b\left(\prod_{N, M} u, g_{N, M}\right)=b\left(u, g_{N, M}\right), \quad \forall g_{N, M} \in P_{N, M}^{0}
$$

The functions $\left\{f_{N, M}\right\}$ which satisfy $(6.24 \mathrm{a})$ have a subsequence, which we again denote by $f_{N, M}$, which is weakly convergent in $V$ using the $b(\cdot, \cdot)$ inner product, to a function $f$. That is, for every $u \in V$

$$
b\left(f_{N, M}, u\right) \rightarrow b(f, u) .
$$

Moreover, the functions $f_{N, M}$ converge strongly to $f$ in $H_{3 / 4}$. That is

$$
\left\|f_{N, M}-f\right\|_{3 / 4} \rightarrow 0
$$


We will show that $T_{N, M} f_{N, M}$ is a Cauchy sequence in $H_{1}$. Let $\min (N, M) \rightarrow \infty$ and $\min \left(N^{\prime}, M^{\prime}\right) \rightarrow \infty$. Consider

$$
\begin{gathered}
R:=\left\|T_{N, M} f_{N, M}-T_{N^{\prime}, M^{\prime}} f_{N^{\prime} M^{\prime}}\right\|_{1} \\
R \leq\left\|T_{N, M} f_{N, M}-T_{N, M} \prod_{N, M} f\right\|_{1}+\left\|T_{N, M} \prod_{N, M} f-T_{N^{\prime}, M^{\prime}} \prod_{N^{\prime}, M^{\prime}} f\right\|_{1} \\
+\left\|T_{N^{\prime}, M^{\prime}} \prod_{N^{\prime}, M^{\prime}} f-T_{N^{\prime}, M^{\prime}} f_{N^{\prime}, M^{\prime}}\right\|_{1}
\end{gathered}
$$

The middle term tends to zero because $T_{N, M}$ is consistent with $T$. Using Lemma 6.4 we have

$$
R \leq K_{T}\left[\left\|f_{N, M}-\prod_{N, M} f\right\|_{3 / 4}+\left\|f_{N^{\prime}, M^{\prime}}-\prod_{N^{\prime}, M^{\prime}} f\right\|_{3 / 4}\right]+\text { middle term }
$$

Since

$$
\left\|f_{N, M}-\prod_{N, M} f\right\|_{3 / 4} \leq\left\|f_{N, M}-f\right\|_{3 / 4}+\left\|f-\prod_{N, M} f\right\|_{1}
$$

we see that $(6.27 \mathrm{~b})$ implies that $R \rightarrow 0$ as $\min \left[\min (N, M), \min \left(N^{\prime}, M^{\prime}\right)\right] \rightarrow \infty$.

The arguments of $[\mathrm{A}],[\mathrm{PW},[\mathrm{G}],[\mathrm{W} 2],[\mathrm{GMP}]$ finally yield our final result.

Theorem 6.3. Let

$$
\hat{S}_{N, M}=\hat{B}_{N, M}^{-1} \hat{A}_{N, M}
$$

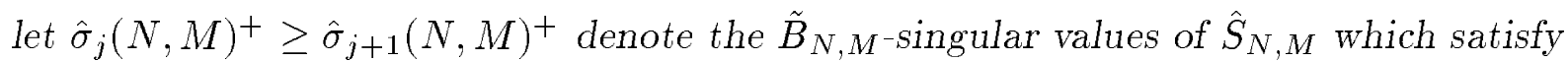

$$
\hat{\sigma}_{j}(N, M)^{+} \geq 1
$$

and let $\hat{\sigma}_{j}(N, M)^{-} \leq \hat{\sigma}_{j+1}(N, M)^{-}$denote the $\tilde{B}_{N, M}$-singular values of $\hat{S}_{N, M}$ which satisfy

$$
0<\hat{\sigma}_{j}(N, M)^{-}<1 .
$$

Then these values "cluster" about $\sigma=1$. Specifically, let $\varepsilon>0$ be given. There is an $N_{1} \geq N_{0}$, depending on $\varepsilon$, and an integer $J$ such that, for $\min (N, M) \geq N_{1}$ we have

$$
\hat{\sigma}_{j}(N, M)^{+}-1<\varepsilon, \quad j \geq J,
$$




$$
1-\hat{\sigma}_{j}(N, M)^{-}<\varepsilon, \quad j \geq J .
$$

These quantities are given by

$$
\begin{gathered}
{\left[\hat{\sigma}_{j}(N, M)^{+}\right]^{2}=\left(\begin{array}{cc}
\max & \min \\
\operatorname{dim} s=j & 0 \neq F \in s
\end{array}\right) \frac{\left(\tilde{B}_{N, M} \hat{S}_{N, M} F, \hat{S}_{N, M} F\right)_{\ell_{2}}}{\left(\tilde{B}_{N, M} F, F\right)_{\ell_{2}}},} \\
{\left[\hat{\sigma}_{j}(N, M)^{-}\right]^{2}=\left(\begin{array}{cc}
\min & \max \\
\operatorname{dim} s=j & 0 \neq F \in s
\end{array}\right) \frac{\left(\tilde{B}_{N, M} \hat{S}_{N, M} F, \hat{S}_{N, M} F\right)_{\ell_{2}}}{\left(\tilde{B}_{N, M} F, F\right)_{\ell_{2}}} .}
\end{gathered}
$$

We close this section with a heuristic discussion of what one might expect of the few $\tilde{B}_{N, M}$-singular values $\hat{\sigma}_{j}^{ \pm}(N, M)$ which are not part of the cluster about $\sigma=1$. Specifically, let

$$
A u=B u-k^{2} u
$$

where the constant $k^{2}$ satisfies

$$
\lambda_{j_{0}}<k^{2}<\lambda_{j_{0}+1}
$$

where $\lambda_{j_{0}}$ and $\lambda_{j_{0}+1}$ are consecutive eigenvalues of the operator $B$. The preconditioner is taken to be $B_{\lambda}$ where

$$
B_{\lambda} u=B u+\lambda u
$$

with

$$
\lambda \geq 0 .
$$

In [GP] the authors consider the problem of choosing $\lambda$ so as to minimize the condition number of $\left[B_{\lambda(N, M)}\right]^{-1} A_{N, M}$. They show that

$$
\lambda=k^{2}
$$

is optimal.

It is easy to see that the $B$-singular values of $B_{\lambda}^{-1} A$ are given by

$$
\frac{\left|\lambda_{j}-k^{2}\right|}{\lambda+\lambda_{j}} \text {. }
$$

Hence, for $(N, M) \geq N_{0}$ we expect

$$
\min \hat{\sigma}_{j}(N, M)^{-} \equiv \min \left\{\frac{\left|\lambda_{j 0}-k^{2}\right|}{\lambda+\lambda_{j 0}}, \frac{\left|\lambda_{j 0+1}-k^{2}\right|}{\lambda+\lambda_{j 0+1}}\right\} .
$$

Thus there is one relatively small $\tilde{B}_{N, M}$-singular value. Moreover, as $k^{2}$ gets very large, this minimal singular value gets quite small.

The largest $\tilde{B}_{N, M}$-singular value will be associated with the smallest eigenvalue $\lambda_{1}$ of $B_{\lambda}$. Thus

$$
\max \sigma_{j}(N, M)^{+} \equiv \frac{k^{2}-\lambda_{1}}{\lambda+\lambda_{1}}
$$

Hence, a large $\lambda$ will make the minimal $\tilde{B}_{N, M}$-singular value small while a smaller $\lambda>0$ increases the maximum $B_{N, M}$-singular value. 


\section{General Preconditioning}

In this section we discuss the matrix

$$
L_{N, M}=\left[\tilde{\beta}_{N, M}\right]^{-1} W_{N, M} \hat{A}_{N, M}
$$

its $\tilde{\beta}_{N, M}$ condition number and the distribution of its $\tilde{\beta}_{N, M}$-singular values.

Let $Q_{N, M}$ be the matrix defined by (5.2). Since $W_{N, M} \hat{B}_{N, M}=\tilde{B}_{N, M}$ is symmetric, the matrix $Q_{N, M}$ is self-adjoint in the $\tilde{\beta}_{N, M}$-innerproduct. Hence Theorem 5.3 gives

Lemma 7.1. For every $U=\left(u_{1}, u_{2}, \cdots, u_{(N+1)(M+1)}\right)^{T}$ we have

$$
\left(\tilde{\beta}_{N, M} Q_{N, M} U, Q_{N, M} U\right)_{\ell_{2}} \sim\left(\tilde{\beta}_{N, M} U, U\right)_{\ell_{2}} .
$$

Theorem 7.1. Let $\min (N, M) \geq N_{0}$. For every $u=\left(u_{1}, u_{2}, \cdots, u_{(N+1)(M+1)}\right)^{T}$ we have

$$
\left(\tilde{\beta}_{N, M}\left[L_{N, M} U\right],\left[L_{N, M} U\right]\right)_{\ell_{2}} \sim\left(\tilde{\beta}_{N, M} U, U\right) \ell_{2} .
$$

This statement (7.3) asserts that these are positive constants, $0<\alpha<\beta$, independent of $(N, M)$, such that the $\tilde{\beta}_{N, M^{-}}$singular values of $L_{N, M}$, denoted by $\sigma_{j}(N, M)$, satisfy

$$
0<\alpha \leq \sigma_{j}(N, M) \leq \beta .
$$

In other words, in the $B$-norm (the $\tilde{\beta}_{N, M}$ in each finite dimensional space) the condition number of $L_{N, M}$ is uniformly bounded.

Proof. We write

$$
L_{N, M}=\left[\tilde{\beta}_{N, M}\right]^{-1}\left[W_{N, M} \hat{B}_{N, M}\right]\left[\hat{B}_{N, M}^{-1} \hat{A}_{N, M}\right] .
$$

That is

$$
L_{N, M}=Q_{N, M} \hat{S}_{N, M}
$$

where $\hat{S}_{N, M}$ is the matrix representation of the operator $S_{N, M}$ defined in (6.3). Let

$$
Z=\hat{S}_{N, M} U
$$

Then

$$
\left(\tilde{\beta}_{N, M}\left[L_{N, M} U\right],\left[L_{N, M} U\right]\right)_{\ell_{2}}=\left(\tilde{\beta}_{N, M}\left[Q_{N, M} Z\right],\left[Q_{N, M} Z\right]\right)_{\ell_{2}} .
$$

Using (7.2) we see that

$$
\begin{gathered}
\left(\tilde{\beta}_{N, M}\left[L_{N, M} U\right],\left[L_{N, M} U\right]\right)_{\ell_{2}} \sim\left(\tilde{\beta}_{N, M} Z, Z\right)_{\ell_{2}} \\
\left(\tilde{\beta}_{N, M}\left[L_{N, M} U\right],\left[L_{N, M} U\right]\right)_{\ell_{2}} \sim\left(\tilde{\beta}_{N, M}\left[\hat{S}_{N, M} U\right],\left[\hat{S}_{N, M} U\right]\right)_{\ell_{2}}
\end{gathered}
$$

Using (6.7c) of Theorem 6.2 we have

$$
\left(\tilde{\beta}_{N, M} Z, Z\right)_{\ell_{2}} \sim\left(\tilde{\beta}_{N, M} U, U\right)_{\ell_{2}}
$$

and hence

$$
\left(\tilde{\beta}_{N, M}\left[L_{N, M} U\right],\left[L_{N, M} U\right]\right)_{\ell_{2}} \sim\left(\tilde{\beta}_{N . M} U, \tilde{\beta}_{N, M} U\right) \ell_{\ell_{2}} .
$$

The theorem is proven.

Finally, we discuss the clustering of the $\sigma_{j}(N, M)$. 
Theorem 7.2. Let $\min (N, M) \geq N_{0}$. Let $\alpha_{0}, \beta_{0}$ be the constants of Theorem 5.3 - given in (5.29b) and (5.29c). Then the $\tilde{\beta}_{N, M}$-singular values of $L_{N, M}, \sigma_{j}(N, M)$, cluster in the interval

$$
\left[\left(\frac{\alpha_{0}^{3}}{\beta_{0}}\right)^{1 / 2},\left(\frac{\beta_{0}^{3}}{\alpha_{0}}\right)^{1 / 2}\right] .
$$

Note: This interval is independent of the operator $A$.

Proof. The $\left[\sigma_{j}(N, M)\right]^{2}$, the squares of the $\tilde{\beta}_{N, M}$-singular values of $L_{N, M}$ are given by the min - max principle applied to the Rayleigh quotient

$$
\rho(U):=\frac{\left(\tilde{\beta}_{N, M}\left[L_{N, M} U\right],\left[L_{N, M} U\right]\right)}{\left(\tilde{\beta}_{N, M} U, U\right)_{\ell_{2}}}
$$

That is, in the notation introduced in the proof of Theorem 7.1,

$$
\rho(U)=\frac{\left(\tilde{\beta}_{N, M}\left[Q_{N, M} Z\right],\left[Q_{N, M} Z\right]\right)_{\ell_{2}}}{\left(\tilde{\beta}_{N, M} U, U\right)_{\ell_{2}}}
$$

Using (5.29b) we have

$$
\alpha_{0}^{2} \frac{\left(\tilde{\beta}_{N, M} Z, Z\right)_{\ell_{2}}}{\left(\tilde{\beta}_{N, M} U, U\right)_{\ell_{2}}} \leq \rho(U) \leq \frac{\beta_{0}^{2}\left(\tilde{\beta}_{N, M} Z, Z\right)_{\ell_{2}}}{\left(\tilde{\beta}_{N, M} U, U\right)_{\ell_{2}}}
$$

Using Theorem 5.3 again we note that

$$
\alpha_{0}\left(\tilde{\beta}_{N, M} Z, Z\right)_{\ell_{2}} \leq\left(\tilde{B}_{N, M} Z, Z\right)_{\ell_{2}} \leq \beta_{0}\left(\tilde{\beta}_{N, M} Z, Z\right)_{\ell_{2}}
$$

Hence, using (7.8) we have

$$
\begin{aligned}
& \frac{\alpha_{0}^{2}}{\beta_{0}} \frac{\left(\tilde{B}_{N, M} Z, Z\right)_{\ell_{2}}}{\left(\tilde{\beta}_{N, M} U, U\right)_{\ell_{2}}} \leq \rho(U) \leq \frac{\beta_{0}^{2}}{\alpha_{0}} \frac{\left(\tilde{B}_{N, M} Z, Z\right)_{\ell_{2}}}{\left(\tilde{\beta}_{N, M} U, U\right)_{\ell_{2}}} \\
& \frac{\alpha_{0}^{3}}{\beta_{0}} \frac{\left(\tilde{B}_{N, M} Z, Z\right)_{\ell_{2}}}{\left(\tilde{B}_{N, M} U, U\right)_{\ell_{2}}} \leq \rho(U) \leq \frac{\beta_{0}^{3}}{\alpha_{0}} \frac{\left(\tilde{B}_{N, M} Z, Z\right)_{\ell_{2}}}{\left(\tilde{B}_{N, M} U, U\right)_{\ell_{2}}} .
\end{aligned}
$$

However the min - max properties of the Rayleigh quotient

$$
\hat{\rho}:=\frac{\left(\tilde{B}_{N, M} Z, Z\right)_{\ell_{2}}}{\left(\tilde{B}_{N, M} U, U\right) \ell_{2}}=\frac{\left(\tilde{B}_{N, M} \hat{S}_{N, M} U, \hat{S}_{N, M} U\right)_{\ell_{2}}}{\left(\tilde{B}_{N, M} U, U\right)_{\ell_{2}}}
$$

are discussed in Theorem 6.3. Its $\min -\max$ values are the quantities $\left[\hat{\sigma}_{j}(N, M)^{ \pm}\right]^{2}$ which cluster about $\sigma=1$. That is, the $\sigma_{j}(N, M)$ satisfy

$$
\frac{\alpha_{0}^{3}}{\beta_{0}}\left[\hat{\sigma}_{j}^{-}(N, M)\right]^{2} \leq\left[\sigma_{j}(N, M)\right]^{2} \leq \frac{\beta_{0}^{3}}{\alpha_{0}}\left[\hat{\sigma}_{j}^{+}(N, M)\right]^{2}
$$

where the $\hat{\sigma}_{j}^{ \pm}(N, M)$ are the $\tilde{B}_{N, M}$-singular values of $\hat{S}_{N, M}$. Thus, the Theorem is proven. 


\section{Piecewise-linear Preconditioning}

The main goal of this section is to establish the estimate (1.14). Then, as indicated in the Introduction, the arguments of Section 7 apply word-for-word to obtain bounds and distribution estimates on the $\tilde{\beta}_{N, M}$-singular values of

$$
L_{N, M}=\tilde{\beta}_{N, M}^{-1} \tilde{A}_{N, M}=\tilde{\beta}_{N, M} W_{N, M} \hat{A}_{N, M}
$$

In this case $\tilde{\beta}_{N, M}$ is the stiffness matrix associated with the finite element discretization of the operator $B$ in the space $Z_{N, M}^{0}$ with the interpolatory basis.

We collect some basic facts which are the result of straightforward, but somewhat tedious, calculations. Let $K$ be the basic rectangle with the four LGL vertices $\left(x_{k}, y_{j}\right),\left(x_{k+1}, y_{j}\right),\left(x_{k+1}, y_{j+1}\right),\left(x_{k}, y_{j+1}\right)$. Let $u(x, y) \in V_{N, M}^{0}$ and on $K$ let

$$
u(x, y)=a+b\left(x-x_{k}\right)+c\left(y-y_{j}\right)+d\left(x-x_{k}\right)\left(y-y_{j}\right), \text { on } K
$$

Let

$$
h:=\left(y_{j+1}-y_{j}\right), \quad m:=\left(x_{k+1}-x_{k}\right)
$$

Then, a direct computation shows that

$$
\begin{gathered}
a=u\left(x_{k}, y_{j}\right), \\
b=\frac{\left[u\left(x_{k+1}, y_{j}\right)-u\left(x_{k}, y_{j}\right)\right]}{m}, \\
c=\frac{\left[u\left(x_{k}, y_{j+1}\right)-u\left(x_{k}, y_{j}\right)\right]}{h}, \\
d=\frac{\left\{\left[u\left(x_{k+1}, y_{j+1}\right)+u\left(x_{k}, y_{j}\right)\right]-\left[u\left(x_{k}, y_{j+1}\right)+u\left(x_{k+1}, y_{j}\right)\right]\right\}}{m h}
\end{gathered}
$$

Let $K_{N, M}$ be the interpolation operator defined by (2.21). Let $w(x, y)=\left(K_{N, M} u\right)(x, y)$. A straightforward computation shows that, on $K$ we have

$$
w(x, y)= \begin{cases}a+b\left(x-x_{k}\right)+c\left(y-y_{j}\right), & (x, y) \in T_{1} \\ \alpha+\beta\left(x-x_{k+1}\right)+\gamma\left(y-y_{j+1}\right), & (x, y) \in T_{2}\end{cases}
$$

where, as in Section 2, $T_{1}$ is the triangle with vertices $\left(x_{k}, y_{j}\right),\left(x_{k+1}, y_{j}\right),\left(x_{k}, y_{j+1}\right)$ and $T_{2}$ is the triangle with vertices $\left(x_{k+1}, y_{j}\right),\left(x_{k+1}, y_{j+1}\right),\left(x_{k}, y_{j+1}\right)$ and

$$
\alpha=a+b m+c h+d m h,
$$




$$
\beta=b+d h,
$$

$$
\gamma=c+d m .
$$

Completing the integrals we find that

$$
\iint_{K} u^{2} d x d y=\frac{A(K)}{9} \mu_{K}^{T}[M(u)] \mu_{K}
$$

where

$$
A(K)=m h
$$

is the area of $K$ and

$$
\mu_{K}:=\left(u\left(x_{k}, y_{j}\right), u\left(x_{k+1}, y_{j}\right), u\left(x_{k+1}, y_{j+1}\right), u\left(x_{k}, y_{j+1}\right)\right)^{T}
$$

is the vector of the four function values which determine $u(x, y)$ on $K$ as well as $w(x, y)$ on $K$ and

$$
M(u):=\left[\begin{array}{cccc}
1 & 1 / 2 & 1 / 4 & 1 / 2 \\
1 / 2 & 1 & 1 / 2 & 1 / 4 \\
1 / 4 & 1 / 2 & 1 & 1 / 2 \\
1 / 2 & 1 / 4 & 1 / 2 & 1
\end{array}\right]
$$

Another integration yields

$$
\iint_{K} w^{2} d x d y=\frac{A(K)}{12} \mu_{K}^{T}[M(w)] \mu_{K}
$$

where

$$
M(w):=\left[\begin{array}{cccc}
1 & 1 / 2 & 0 & 1 / 2 \\
1 / 2 & 2 & 1 / 2 & 1 \\
0 & 1 / 2 & 1 & 1 / 2 \\
1 / 2 & 1 & 1 / 2 & 2
\end{array}\right]
$$

Lemma 8.1. There are two positive constants, $0<\alpha<\beta$, such that, for each $u(x, y) \in$ $V_{N, M}^{0}$, we have

$$
\alpha\|u\|_{L_{2}}^{2} \leq\left\|K_{N, M} u\right\|_{L_{2}}^{2} \leq \beta\|u\|_{L_{2}}^{2}
$$

The constants $\alpha$ and $\beta$ are given by

$$
\alpha \geq .19=\alpha_{0}
$$




$$
\beta \leq 10.5=\beta_{0}
$$

Proof. A calculation using Matlab gives the eigenvalues of $M(u)$ and $M(w)$. These are:

The eigenvalues of $M(u)$ are

$$
1 / 4,3 / 4,3 / 4,9 / 4 . \quad[M(u)]
$$

the eigenvalues of $M(w)$ are

$$
\lambda_{1} \sim 0.5858,1,1, \lambda_{4} \sim 3.4142 . \quad[M(w)]
$$

Thus

$$
\lambda_{1} \geq 0.58, \quad \lambda_{4} \leq 3.5
$$

Using (8.7) and (8.9) we see that: for each rectangle $K$, we have

$$
\frac{9}{12}\left[\frac{0.5858}{9 / 4}\right] \iint_{K} u^{2} d x d y \leq \iint_{K} w^{2} d x d y .
$$

That is,

$$
\alpha_{0} \iint_{K} u^{2} d x d y \leq \iint_{K}\left|K_{N, M} u\right|^{2} d x d y
$$

Similarly,

$$
\iint_{K} w^{2} d x d y \leq \frac{3.5}{1 / 4} \cdot \frac{9}{12} \iint_{K} u^{2} d x d y
$$

That is,

$$
\iint_{K}\left|K_{N, M} u\right|^{2} d x d y \leq \beta_{0} \iint_{K} u^{2} d x d y .
$$

The final result follows upon summation over the rectangles $K$.

In dealing with the integrals

$$
\iint_{K}|\nabla u|^{2} d x d y, \quad \iint_{K}|\nabla w|^{2} d x d y
$$

we find it convenient to use the vector

$$
\delta_{K}:=(b, c, d)^{T}
$$

Using these variables rather than $\mu_{K}$ enables us to bypass the difficulties caused by the constant functions. 
Further elementary, but tedious, calculations show that

$$
\iint_{K}|\nabla u|^{2} d x d y=A(K) \delta_{K}^{T} S_{K}(u) \delta_{K}
$$

where

$$
S_{K}(u):=\left[\begin{array}{ccc}
1 & 0 & h / 2 \\
0 & 1 & m / 2 \\
h / 2 & m / 2 & \left(m^{2}+h^{2}\right) / 3
\end{array}\right]
$$

and

$$
\iint_{K}|\nabla w|^{2} d x d y=A(K) \delta_{K}^{T} S_{K}(w) \delta_{K}
$$

where

$$
S_{K}(w)=\left[\begin{array}{ccc}
1 & 0 & h / 2 \\
0 & 1 & m / 2 \\
h / 2 & m / 2 & \left(h^{2}+m^{2}\right) / 2
\end{array}\right] .
$$

Lemma 8.2. Let $u \in V_{N, M}^{0}$. Then

$$
\iint_{\Omega}|\nabla u|^{2} d x d y \leq \iint_{\Omega}\left|\nabla\left(K_{N, M} u\right)\right|^{2} \leq 3 \iint_{\Omega}|\nabla u|^{2} d x d y
$$

Proof. Once more, it suffices to establish these inequalities for each rectangle $K$. Clearly

$$
S_{K}(w)=S_{K}(u)+1 / 6\left[\begin{array}{ccc}
0 & 0 & 0 \\
0 & 0 & 0 \\
0 & 0 & \left(h^{2}+m^{2}\right)
\end{array}\right] .
$$

Hence, using (8.17) and (8.18) we have

$$
\iint_{K}|\nabla u|^{2} d x d y \leq \iint_{K}\left|\nabla\left(K_{N, M} u\right)\right|^{2} d x d y .
$$

Similarly, we verify that

$$
S_{K}(w)=3 S_{K}(u)-2 \rho_{K}
$$

where

$$
\rho_{K}:=\left[\begin{array}{ccc}
1 & 0 & h / 2 \\
0 & 1 & m / 2 \\
h / 2 & m / 2 & \left(h^{2}+m^{2}\right) / 4
\end{array}\right] .
$$


A simple computation shows that

$$
\operatorname{det} \rho_{K}=0 .
$$

Further computation shows that $\rho_{K}$ is positive semi-definite. Hence

$$
\iint_{K}\left|\nabla\left(K_{M, N} u\right)\right|^{2} d x d y \leq 3 \iint_{K}|\nabla U|^{2} d x d y
$$

and the Lemma is proven.

Theorem 8.1. Let $\alpha_{0}$ and $\beta_{0}$ be the constants of Lemma 8.1. Then, for all $u \in V_{N, M}^{0}$ we have

$$
\left(\alpha_{0}\right)^{1 / 2}\|u\|_{1} \leq\left\|K_{N, M} u\right\|_{1} \leq\left(\beta_{0}\right)^{1 / 2}\|u\|_{1} .
$$

Proof. The Theorem follows immediately from the two preceding Lemmas.

Having established this result we immediately obtain the $Z_{N, M}^{0}$ version of Theorem 5.3, Theorem 7.1 and Theorem 7.2 .

Theorem 8.2. Let $\tilde{\beta}_{N, M}$ be the stiffness matrix of the finite element discretization of the operator $B$ in the space $Z_{N, M}^{0}$ with the interpolatory basis (i.e., the degrees of freedom are the values of $w(x, y) \in Z_{N, M}^{0}$ at the 2 dimensional $L G L$ points). For every $U=\left(u_{1}, u_{2}, \cdots, u_{(N+1)(M+1)}\right)^{T}$ we have

$$
\left(\tilde{\beta}_{N, M} U, U\right)_{\ell_{2}} \sim\left(\tilde{B}_{N, M} U, U\right)_{\ell^{2}}
$$

This statement is equivalent to: Let $0<q_{1} \leq \cdots \leq q_{(N+1)(M+1)}$ be the eigenvalues of the matrix

$$
Q_{N, M}=\tilde{\beta}_{N, M}^{-1} W_{N, M} \hat{B}_{N, M}=\tilde{\beta}_{N, M}^{-1} \tilde{B}_{N, M} .
$$

There are constants $0<\alpha_{1}<\beta_{1}$ such that

$$
0<\alpha_{1} \leq q_{j} \leq \beta_{1} .
$$

Theorem 8.3. Let $\tilde{\beta}_{N, M}$ be as in Theorem 8.2. Let $\min (N, M) \geq N_{0}$. For every $U=$ $\left(u_{1}, u_{2}, \cdots, u_{(N+1)(M+1)}\right)^{T}$, we have

$$
\left(\tilde{\beta}_{N, M}\left[L_{N, M} U\right],\left[L_{N, M} U\right]\right)_{\ell_{2}} \sim\left(\tilde{\beta}_{N, M} U, U\right) \ell_{2} .
$$

This statement (8.27) asserts that these are positive constants $0<\alpha<\beta$, independent of $(N, M)$, such that the $\tilde{\beta}_{N, M}$-singular values of $L_{N, M}$, denoted by $\sigma_{j}(N, M)$, satisfy

$$
0<\alpha \leq \sigma_{j}(N, M) \leq \beta .
$$

In other words, in the $B$-norm, the condition number of $L_{N, M}$ is uniformly bounded.

Theorem 8.4. Let $\min (N, M) \geq N_{0}$. Let $\alpha_{1}, \beta_{1}$ be the constants of (8.26). Then the $\beta_{N, M}$ - singular values of $L_{N, M}$, the $\sigma_{j}(N, M)$, cluster in the interval

$$
\left[\left(\frac{\alpha_{1}^{3}}{\beta_{1}}\right)^{1 / 2},\left(\frac{\beta_{1}^{3}}{\alpha_{1}}\right)^{1 / 2}\right] .
$$




\section{Computational Results}

In this section we discuss numerical experiments of both one-and-two-dimensional problems. First, let us consider the following second-order elliptic problem in one dimension:

$$
A u:=-u_{x x}+s u_{x}+b u \quad \text { for } x \in(-1,1)
$$

with boundary conditions

$$
u_{x}(-1)-u(-1)=0=u(1)
$$

Let $B$ be the operator defined by

$$
B v=-v_{x x}+|b| v \text { for } x \in(-1,1)
$$

with boundary conditions

$$
v_{x}(-1)=0=v(1)
$$

We let $A_{N}$ be the pseudospectral discretization based on the the Legendre-Gauss-Lobatto [LGL] points representation of the operator $A$. We consider the we weak formulation which leads to the following treatment of the Robin boundary condition:

$$
\begin{gathered}
-w_{0}\left(u_{x x}^{N}(-1)+s u_{x}^{N}(-1)+b u^{N}(-1)\right)-u_{x}^{N}(-1)=w_{0} f(-1) \\
-u_{x x}^{N}\left(x_{j}\right)+s u_{x}^{N}\left(x_{j}\right)+b u^{N}\left(x_{j}\right)=f\left(x_{j}\right), \text { for } j=1,2, \ldots, N-1 \\
u^{N}(1)=0,
\end{gathered}
$$

where $w_{0}=2 /(N(N+1))$. Consider the linear equation

$$
\hat{A}_{N} u=f
$$

which arises in the numerical solution of the boundary value problem

$$
A u=f
$$

We let $\tilde{\beta}_{N}$ be the finite-element stiffness matrix associated with the operator $B$ in the finite element space $V_{N}^{0}$. We precondition $\hat{A}_{N}$ with $\tilde{\beta}_{N} W_{N}$, where $W_{N}$ is a diagonal matrix whose entries are the Legendre-Gauss-Lobatto quadrature weights. Finally, let $L_{N}$ denote the preconditioned matrix given by

$$
L_{N}=\tilde{\beta}_{N}^{-1} W_{N} \hat{A}_{N} .
$$


We present numerical results for two problems: one in which $A$ has all positive eigenvalues, the other in which $A$ has a few negative eigenvalues. The problems presented here are representative of similar problems for which we did numerical experiments. The eigenvalues listed are actually (for the indefinite cases) the absolute values of the eigenvalues. The $\beta$ singular values of $L_{N}$ are defined, as in Section 2, as the square roots of the eigenvalues of the matrix $\left[\tilde{\beta}_{N}^{-1}\left(W_{N} \hat{A}_{N}\right)^{*}\right]\left[\tilde{\beta}_{N}^{-1}\left(W_{N} A\right)\right]$. The singular values of $L_{N}$ are, as usual, the square roots of the eigenvalues of the matrix $L_{N}^{*} L_{N}$. One can see from these tables that the eigenvalues, singular values, and $\beta$-singular values are clustered and produce small condition numbers.

For the case in which $A$ is positive definite, we take $s=5, b=\left(\left(\frac{5}{4}\right) \pi\right)^{2}$. We report the eigenvalues, singular values, and $\beta$-singular values for various values of $N$ along with the associated condition numbers in tables $9.1-9.3$.

Table 9.1

Extreme Eigenvalues w/ spectral condition nos: $L_{N}, b=\left(\frac{5}{4} \pi\right)^{2}, s=5$.

\begin{tabular}{|r|c|c|c|}
\hline \multicolumn{1}{|c|}{$N$} & min. eig. & max. eig. & condition no. \\
\hline 8 & $0.1167964 \mathrm{E}+01$ & $0.1847266 \mathrm{E}+01$ & $0.1581612 \mathrm{E}+01$ \\
16 & $0.1130649 \mathrm{E}+01$ & $0.1991085 \mathrm{E}+01$ & $0.1761010 \mathrm{E}+01$ \\
32 & $0.1107615 \mathrm{E}+01$ & $0.2185080 \mathrm{E}+01$ & $0.1972780 \mathrm{E}+01$ \\
64 & $0.1064111 \mathrm{E}+01$ & $0.2315478 \mathrm{E}+01$ & $0.2175973 \mathrm{E}+01$ \\
128 & $0.1033620 \mathrm{E}+01$ & $0.2388818 \mathrm{E}+01$ & $0.2311117 \mathrm{E}+01$ \\
256 & $0.1017152 \mathrm{E}+01$ & $0.2427464 \mathrm{E}+01$ & $0.2386530 \mathrm{E}+01$ \\
512 & $0.1008628 \mathrm{E}+01$ & $0.2447273 \mathrm{E}+01$ & $0.2426340 \mathrm{E}+01$ \\
1024 & $0.1004330 \mathrm{E}+01$ & $0.2457297 \mathrm{E}+01$ & $0.2446703 \mathrm{E}+01$ \\
\hline
\end{tabular}

Table 9.2

\begin{tabular}{|r|c|c|c|}
\multicolumn{1}{|c|}{ Extreme singular values $w /$ condition nos: $L_{N}, b=\left(\frac{5}{4} \pi\right)^{2}, s=5}$. \\
\hline \multicolumn{1}{|c|}{$N$} & min. sing. & max. sing. & condition no. \\
\hline 8 & $0.4483755 \mathrm{E}+00$ & $0.2268380 \mathrm{E}+01$ & $0.5059109 \mathrm{E}+01$ \\
36 & $0.3372913 \mathrm{E}+00$ & $0.2314083 \mathrm{E}+01$ & $0.6860783 \mathrm{E}+01$ \\
64 & $0.2626978 \mathrm{E}+00$ & $0.2657845 \mathrm{E}+01$ & $0.1011750 \mathrm{E}+02$ \\
128 & $0.2001321 \mathrm{E}+00$ & $0.3439328 \mathrm{E}+01$ & $0.1718529 \mathrm{E}+02$ \\
256 & $0.1482101 \mathrm{E}+00$ & $0.4630036 \mathrm{E}+01$ & $0.3123968 \mathrm{E}+02$ \\
512 & $0.7709549 \mathrm{E}-01$ & $0.8895498 \mathrm{E}+01$ & $0.1153829 \mathrm{E}+03$ \\
1024 & $0.5490092 \mathrm{E}-01$ & $0.1249217 \mathrm{E}+02$ & $0.2275402 \mathrm{E}+03$ \\
\hline
\end{tabular}


Table 9.3

Extreme $\beta$-singular values $w /$ condition nos: $L_{N}, b=\left(\frac{5}{4} \pi\right)^{2}, s=5$.

\begin{tabular}{|r|c|c|c|}
\hline \multicolumn{1}{|c|}{$N$} & min. $\beta$-sing. & max. $\beta$-sing. & condition no. \\
\hline 8 & $0.7090087 \mathrm{E}+00$ & $0.2202798 \mathrm{E}+01$ & $0.3106870 \mathrm{E}+01$ \\
16 & $0.7162984 \mathrm{E}+00$ & $0.2254583 \mathrm{E}+01$ & $0.3147547 \mathrm{E}+01$ \\
32 & $0.7177499 \mathrm{E}+00$ & $0.2337237 \mathrm{E}+01$ & $0.3256339 \mathrm{E}+01$ \\
64 & $0.7180715 \mathrm{E}+00$ & $0.2396285 \mathrm{E}+01$ & $0.3337112 \mathrm{E}+01$ \\
128 & $0.7181492 \mathrm{E}+00$ & $0.2430340 \mathrm{E}+01$ & $0.3384172 \mathrm{E}+01$ \\
256 & $0.7181685 \mathrm{E}+00$ & $0.2448497 \mathrm{E}+01$ & $0.3409363 \mathrm{E}+01$ \\
512 & $0.7181734 \mathrm{E}+00$ & $0.2457856 \mathrm{E}+01$ & $0.3422371 \mathrm{E}+01$ \\
1024 & $0.7181746 \mathrm{E}+00$ & $0.2462605 \mathrm{E}+01$ & $0.3428979 \mathrm{E}+01$ \\
\hline
\end{tabular}

For the case of the indefinite problem we take $s=5, b=-\left(\frac{5}{4} \pi\right)^{2}$. One can find by elementary methods that the operator $A$ has 2 negative eigenvalues in this case. We also computed the number of negative eigenvalues of $A_{N}$ and it too had 2 negative eigenvalues (at least for $N=8,16, \ldots, 1024)$. We report the extreme eigenvalues, singular values and $\beta$-singular values along with associated condition numbers for these values of $N$ in Tables 9.4-9.6.

Table 9.4

Extreme Eigenvalues w/ spectral condition nos: $\left.L_{N}, b=-\left(\frac{5}{4}\right) \pi\right)^{2}, s=5$.

\begin{tabular}{|r|c|c|c|}
\hline \multicolumn{1}{|c|}{$N$} & min. eig & max. eig & condition no. \\
\hline 8 & $0.1998642 \mathrm{E}+00$ & $0.1129986 \mathrm{E}+01$ & $0.5653770 \mathrm{E}+01$ \\
16 & $0.2034377 \mathrm{E}+00$ & $0.1789578 \mathrm{E}+01$ & $0.8796686 \mathrm{E}+01$ \\
32 & $0.2080575 \mathrm{E}+00$ & $0.2133634 \mathrm{E}+01$ & $0.1025502 \mathrm{E}+02$ \\
64 & $0.2096394 \mathrm{E}+00$ & $0.2302579 \mathrm{E}+01$ & $0.1098352 \mathrm{E}+02$ \\
128 & $0.2100719 \mathrm{E}+00$ & $0.2385592 \mathrm{E}+01$ & $0.1135607 \mathrm{E}+02$ \\
256 & $0.2101830 \mathrm{E}+00$ & $0.2426658 \mathrm{E}+01$ & $0.1154545 \mathrm{E}+02$ \\
512 & $0.2102110 \mathrm{E}+00$ & $0.2447071 \mathrm{E}+01$ & $0.1164102 \mathrm{E}+02$ \\
1024 & $0.2102181 \mathrm{E}+00$ & $0.2457247 \mathrm{E}+01$ & $0.1168904 \mathrm{E}+02$ \\
\hline
\end{tabular}

Table 9.5

Extreme singular values $w /$ condition nos: $L_{N}, b=-\left(\frac{5}{4} \pi\right)^{2}, s=5$.

\begin{tabular}{|r|c|c|c|}
\hline \multicolumn{1}{|c|}{$N$} & min. sing. & max. sing. & condition no. \\
\hline 8 & $0.9485507 \mathrm{E}-02$ & $0.1691159 \mathrm{E}+01$ & $0.1782887 \mathrm{E}+03$ \\
16 & $0.7362417 \mathrm{E}-02$ & $0.2126797 \mathrm{E}+01$ & $0.2888722 \mathrm{E}+03$ \\
32 & $0.5552403 \mathrm{E}-02$ & $0.2333357 \mathrm{E}+01$ & $0.4202428 \mathrm{E}+03$ \\
64 & $0.4062287 \mathrm{E}-02$ & $0.3130882 \mathrm{E}+01$ & $0.7707192 \mathrm{E}+03$ \\
128 & $0.2921448 \mathrm{E}-02$ & $0.4398256 \mathrm{E}+01$ & $0.1505506 \mathrm{E}+04$ \\
256 & $0.2083042 \mathrm{E}-02$ & $0.6208273 \mathrm{E}+01$ & $0.2980388 \mathrm{E}+04$ \\
512 & $0.1479009 \mathrm{E}-02$ & $0.8774315 \mathrm{E}+01$ & $0.5932563 \mathrm{E}+04$ \\
1024 & $0.1047958 \mathrm{E}-02$ & $0.1240583 \mathrm{E}+02$ & $0.1183809 \mathrm{E}+05$ \\
\hline
\end{tabular}


Table 9.6

Extreme $\beta$-singular values $w /$ condition nos: $L_{N}, b=-\left(\frac{5}{4} \pi\right)^{2}, s=5$.

\begin{tabular}{|r|c|c|c|}
\hline \multicolumn{1}{|c|}{$N$} & min. $\beta$-sing. & max. $\beta$-sing. & condition no. \\
\hline 8 & $0.7998722 \mathrm{E}-02$ & $0.1533053 \mathrm{E}+01$ & $0.1916622 \mathrm{E}+03$ \\
16 & $0.7616712 \mathrm{E}-02$ & $0.2053065 \mathrm{E}+01$ & $0.2695474 \mathrm{E}+03$ \\
32 & $0.7514645 \mathrm{E}-02$ & $0.2283756 \mathrm{E}+01$ & $0.3039074 \mathrm{E}+03$ \\
64 & $0.7488212 \mathrm{E}-02$ & $0.2382606 \mathrm{E}+01$ & $0.3181809 \mathrm{E}+03$ \\
128 & $0.7481484 \mathrm{E}-02$ & $0.2426887 \mathrm{E}+01$ & $0.3243858 \mathrm{E}+03$ \\
256 & $0.7479787 \mathrm{E}-02$ & $0.2447630 \mathrm{E}+01$ & $0.3272325 \mathrm{E}+03$ \\
512 & $0.7479360 \mathrm{E}-02$ & $0.2457639 \mathrm{E}+01$ & $0.3285894 \mathrm{E}+03$ \\
1024 & $0.7479250 \mathrm{E}-02$ & $0.2462551 \mathrm{E}+01$ & $0.3292510 \mathrm{E}+03$ \\
\hline
\end{tabular}

It may appear from these tables that the preconditioning is not very effective. However, this is not the case in light of Theorem 7.2 which guarantees that the $\beta$-singular values will be nicely clustered. This suggests that conjugate gradient can be applied to the normal equations using the $\beta$-inner product. Numerical evidence presented in Table 9.7 confirms this. Also, numerical results on the singular values presented in Table 9.8 suggests that the singular values are nicely clustered. At present we do not have a proof of this. However, this empirical evidence suggests that one can successfully apply conjugate gradient using the $l_{2}$-inner product. In fact, in both cases ( $\beta$-singular and singular values), only one value is outside the appropriate interval (for all $N=8,16, \ldots, 1024$ ). Condition numbers in Tables 9.7 and 9.8 are based on all $\beta$-singular and singular values excluding the largest and smallest. Actually, one can obtain almost identical results by excluding only the smallest values.

Table 9.7

2nd Extreme singular values $w /$ condition nos: $L_{N}, b=-\left(\frac{5}{4} \pi\right)^{2}, s=5$.

\begin{tabular}{|r|c|c|c|}
\hline \multicolumn{1}{|c|}{$N$} & 2nd min. sing. & 2nd max. sing. & condition no. \\
\hline 8 & $0.8036099 \mathrm{E}+00$ & $0.1433090 \mathrm{E}+01$ & $0.1783315 \mathrm{E}+01$ \\
16 & $0.6747487 \mathrm{E}+00$ & $0.1906044 \mathrm{E}+01$ & $0.2824820 \mathrm{E}+01$ \\
32 & $0.6100785 \mathrm{E}+00$ & $0.2258877 \mathrm{E}+01$ & $0.3702600 \mathrm{E}+01$ \\
64 & $0.5818087 \mathrm{E}+00$ & $0.2395103 \mathrm{E}+01$ & $0.4116651 \mathrm{E}+01$ \\
128 & $0.5688084 \mathrm{E}+00$ & $0.2433109 \mathrm{E}+01$ & $0.4277554 \mathrm{E}+01$ \\
256 & $0.5626150 \mathrm{E}+00$ & $0.2450706 \mathrm{E}+01$ & $0.4355920 \mathrm{E}+01$ \\
512 & $0.5595988 \mathrm{E}+00$ & $0.2459166 \mathrm{E}+01$ & $0.4394517 \mathrm{E}+01$ \\
1024 & $0.5581114 \mathrm{E}+00$ & $0.2463312 \mathrm{E}+01$ & $0.4413656 \mathrm{E}+01$ \\
\hline
\end{tabular}


Table 9.8

2nd Extreme $\beta$-singular values $w /$ condition nos: $L_{N}, b=-\left(\frac{5}{4} \pi\right)^{2}, s=5$.

\begin{tabular}{|r|c|c|c|}
\hline \multicolumn{1}{|c|}{$N$} & 2nd min. $\beta$-sing. & 2nd max. $\beta$-sing. & condition no. \\
\hline 8 & $0.8516556 \mathrm{E}+00$ & $0.1486775 \mathrm{E}+01$ & $0.1745747 \mathrm{E}+01$ \\
16 & $0.7354986 \mathrm{E}+00$ & $0.1919885 \mathrm{E}+01$ & $0.2610318 \mathrm{E}+01$ \\
32 & $0.7002824 \mathrm{E}+00$ & $0.2177841 \mathrm{E}+01$ & $0.3109946 \mathrm{E}+01$ \\
64 & $0.6906184 \mathrm{E}+00$ & $0.2318123 \mathrm{E}+01$ & $0.3356590 \mathrm{E}+01$ \\
128 & $0.6881112 \mathrm{E}+00$ & $0.2391556 \mathrm{E}+01$ & $0.3475537 \mathrm{E}+01$ \\
256 & $0.6874753 \mathrm{E}+00$ & $0.2429167 \mathrm{E}+01$ & $0.3533460 \mathrm{E}+01$ \\
512 & $0.6873154 \mathrm{E}+00$ & $0.2448205 \mathrm{E}+01$ & $0.3561981 \mathrm{E}+01$ \\
1024 & $0.6872753 \mathrm{E}+00$ & $0.2457783 \mathrm{E}+01$ & $0.3576126 \mathrm{E}+01$ \\
\hline
\end{tabular}

These problems are representative of other problems we tested including problems with pure Dirichlet boundary conditions. 
We now turn our attention to two-dimensional problems. Let $\Omega$ be the square $[-1,1] \times$ $[-1,1]$ and consider a uniformly elliptic operator given by

$$
A u:=-\left[u_{x x}+u_{y y}\right]+a_{1}\left(u_{x}+u_{y}\right)+a_{0} u \text { in } \Omega
$$

with boundary conditions

$$
u=0 \text { on } \Gamma_{0},
$$

where

$$
\partial \Omega=\Gamma_{0}
$$

and $a_{0}<0$. We assume that $A$ is an invertible operator, but not necessarily definite. Let $\left\{A_{N, M}\right\}$ be a family of spectral collocation discretizations based on the Legendre-GaussLobatto [LGL] points representation of the operator $A$. Consider the systems of linear equations.

$$
\hat{A}_{N, M} U=F
$$

which arise in the numerical solution of the boundary value problem

$$
A u=f
$$

using these spectral collocation discretizations and the Lagrange basis $\left\{\varphi_{i j}(x, y)\right\}$ of the polynomial space $P_{N, M}^{0}$ (see section 2 for a complete discussion of notations etc). Let $\tilde{\beta}_{N, M}$ be the stiffness matrix of the finite element discretization. We precondition (9.2) as

$$
\tilde{\beta}_{N, M}^{-1} W_{N, M} \hat{A}_{N, M} U=\tilde{\beta}_{N, M}^{-1} W_{N, M} F,
$$

where $W_{N, M}$ is the diagonal matrix of the quadrative weights $\omega_{k} \hat{\omega}_{j}$ associated with the Gauss-Lobatto quadrature. $\tilde{\beta}_{N, M}$ is the stiffness matrix of the symmetric positive definite operator of the form

$$
B v=-\left[v_{x x}+v_{y y}\right]+b v \text { in } \Omega
$$

with boundary conditions

$$
u=0 \text { on } \Gamma_{0},
$$

where

$$
\partial \Omega=\Gamma_{0} .
$$

The finite element space employed here is the space of continuous piecewise bilinear functions, $V_{N, M}^{0}$, with the basis being the tensor product of the one dimensional "hat" functions. 
One can show that the eigenvalues of the operator in (9.6) are given by

$$
\left(\lambda_{k, j}+a_{0}\right),
$$

where

$$
\lambda_{k, j}=\frac{1}{4}\left(2 a_{1}^{2}+\pi^{2}\left(j^{2}+k^{2}\right)\right),
$$

and $k, j=1,2, \ldots$. Clearly, $A$ has negative eigenvalues for values of $k, j$ such that

$$
\left|\lambda_{k, j}\right|<\left|a_{0}\right| .
$$

We report computational results for two cases which are representative of those which have negative eigenvalues (i.e., are indefinite), but are nonsingular. For the case of $a_{0}=-34.05$, and $a_{1}=6$, the operator $A$ has 3 negative eigenvalues and its minimum eigenvalue is 3.68920880217871527 . For $a_{0}=-22.1725$, and $a_{1}=3.5, A$ has 3 negative eigenvalues and its minimum eigenvalue is 3.69170880217871655 . For comparison we show the number of negative eigenvalues and the minimum eigenvalue of $A_{N, N}$, the pseudospectral approximation to $A$, in Tables 9.9 and 9.10. (In our numerical examples, we take $N=M$.) This comparison serves as a measure of how good an approximation $A_{N, N}$ is to $A$.

\section{Table 9.9}

Number of negative eigenvalues and minimum eigenvalues of $A_{N, N}$, with $a_{0}=-34.05, a_{1}=6$

\begin{tabular}{|r|r|c|}
\hline$(N-1)^{2}$ & No. neg. eig. & Min. eig. \\
\hline 49 & 3 & $0.3696983 \mathrm{E}+01$ \\
81 & 3 & $0.3689242 \mathrm{E}+01$ \\
121 & 3 & $0.3689209 \mathrm{E}+01$ \\
169 & 3 & $0.3689209 \mathrm{E}+01$ \\
225 & 3 & $0.3689209 \mathrm{E}+01$ \\
289 & 3 & $0.3689209 \mathrm{E}+01$ \\
361 & 3 & $0.3689209 \mathrm{E}+01$ \\
441 & 3 & $0.3689209 \mathrm{E}+01$ \\
529 & 3 & $0.3689209 \mathrm{E}+01$ \\
625 & 3 & $0.3689209 \mathrm{E}+01$ \\
729 & 3 & $0.3689209 \mathrm{E}+01$ \\
841 & 3 & $0.3689209 \mathrm{E}+01$ \\
961 & 3 & $0.3689209 \mathrm{E}+01$ \\
1089 & 3 & $0.3689209 \mathrm{E}+01$ \\
1225 & 3 & $0.3689209 \mathrm{E}+01$ \\
1369 & 3 & $0.3689209 \mathrm{E}+01$ \\
1521 & 3 & $0.3689209 \mathrm{E}+01$ \\
2401 & 3 & $0.3689209 \mathrm{E}+01$ \\
\hline
\end{tabular}


Table 9.10

Number of negative eigenvalues and minimum eigenvalues of $A_{N, N}$, with $a_{0}=-22.1725, a_{1}=3.5$

\begin{tabular}{|r|r|c|}
\hline$(N-1)^{2}$ & No. neg. eig. & Min. eig. \\
\hline 49 & 3 & $0.3691910 \mathrm{E}+01$ \\
81 & 3 & $0.3691710 \mathrm{E}+01$ \\
121 & 3 & $0.3691709 \mathrm{E}+01$ \\
169 & 3 & $0.3691709 \mathrm{E}+01$ \\
225 & 3 & $0.3691709 \mathrm{E}+01$ \\
289 & 3 & $0.3691709 \mathrm{E}+01$ \\
361 & 3 & $0.3691709 \mathrm{E}+01$ \\
441 & 3 & $0.3691709 \mathrm{E}+01$ \\
529 & 3 & $0.3691709 \mathrm{E}+01$ \\
625 & 3 & $0.3691709 \mathrm{E}+01$ \\
729 & 3 & $0.3691709 \mathrm{E}+01$ \\
841 & 3 & $0.3691709 \mathrm{E}+01$ \\
961 & 3 & $0.3691709 \mathrm{E}+01$ \\
1089 & 3 & $0.3691709 \mathrm{E}+01$ \\
1225 & 3 & $0.3691709 \mathrm{E}+01$ \\
1369 & 3 & $0.3691709 \mathrm{E}+01$ \\
1521 & 3 & $0.3691709 \mathrm{E}+01$ \\
2401 & 3 & $0.3691709 \mathrm{E}+01$ \\
\hline
\end{tabular}

The eigenvalues, singular and $\beta_{N, N}$-singular values of preconditioned matrices $L_{N, N}$ for these two cases are exhibited in the Tables 9.11-9.16. 
Table 9.11

Extreme Eigenvalues w/ spectral condition nos: $L_{N, N}, a_{0}=-34.05, a_{1}=6$

\begin{tabular}{|r|c|c|c|}
\hline$N$ & min. eig. & max eig. & condition no. \\
\hline 8 & $0.1013053 \mathrm{E}+00$ & $0.3208008 \mathrm{E}+01$ & $0.3166672 \mathrm{E}+02$ \\
10 & $0.1004212 \mathrm{E}+00$ & $0.3951367 \mathrm{E}+01$ & $0.3934793 \mathrm{E}+02$ \\
12 & $0.1015879 \mathrm{E}+00$ & $0.4495625 \mathrm{E}+01$ & $0.4425354 \mathrm{E}+02$ \\
14 & $0.1029258 \mathrm{E}+00$ & $0.4901123 \mathrm{E}+01$ & $0.4761803 \mathrm{E}+02$ \\
16 & $0.1040905 \mathrm{E}+00$ & $0.5211775 \mathrm{E}+01$ & $0.5006967 \mathrm{E}+02$ \\
18 & $0.1050387 \mathrm{E}+00$ & $0.5456174 \mathrm{E}+01$ & $0.5194440 \mathrm{E}+02$ \\
20 & $0.1057977 \mathrm{E}+00$ & $0.5652912 \mathrm{E}+01$ & $0.5343133 \mathrm{E}+02$ \\
22 & $0.1064051 \mathrm{E}+00$ & $0.5814396 \mathrm{E}+01$ & $0.5464395 \mathrm{E}+02$ \\
24 & $0.1068945 \mathrm{E}+00$ & $0.5949156 \mathrm{E}+01$ & $0.5565446 \mathrm{E}+02$ \\
26 & $0.1072923 \mathrm{E}+00$ & $0.6063216 \mathrm{E}+01$ & $0.5651118 \mathrm{E}+02$ \\
28 & $0.1076189 \mathrm{E}+00$ & $0.6160944 \mathrm{E}+01$ & $0.5724778 \mathrm{E}+02$ \\
30 & $0.1078896 \mathrm{E}+00$ & $0.6245570 \mathrm{E}+01$ & $0.5788851 \mathrm{E}+02$ \\
32 & $0.1081161 \mathrm{E}+00$ & $0.6319536 \mathrm{E}+01$ & $0.5845137 \mathrm{E}+02$ \\
34 & $0.1083073 \mathrm{E}+00$ & $0.6384718 \mathrm{E}+01$ & $0.5895003 \mathrm{E}+02$ \\
36 & $0.1084699 \mathrm{E}+00$ & $0.6442578 \mathrm{E}+01$ & $0.5939506 \mathrm{E}+02$ \\
38 & $0.1086094 \mathrm{E}+00$ & $0.6494275 \mathrm{E}+01$ & $0.5979480 \mathrm{E}+02$ \\
40 & $0.1087297 \mathrm{E}+00$ & $0.6540736 \mathrm{E}+01$ & $0.6015592 \mathrm{E}+02$ \\
50 & $0.1091398 \mathrm{E}+00$ & $0.6716600 \mathrm{E}+01$ & $0.6154125 \mathrm{E}+02$
\end{tabular}

Table 9.12

Extreme singular values $w /$ condition nos: $L_{N, N}, a_{0}=-34.05, a_{1}=6$

\begin{tabular}{|r|c|c|c|}
\hline$N$ & min. sing. & max. sing. & condition no. \\
\hline 8 & $0.1271260 \mathrm{E}-03$ & $0.4391974 \mathrm{E}+01$ & $0.3454819 \mathrm{E}+05$ \\
10 & $0.1190179 \mathrm{E}-03$ & $0.5107123 \mathrm{E}+01$ & $0.4291056 \mathrm{E}+05$ \\
12 & $0.1138260 \mathrm{E}-03$ & $0.5566465 \mathrm{E}+01$ & $0.4890330 \mathrm{E}+05$ \\
14 & $0.1107207 \mathrm{E}-03$ & $0.5883391 \mathrm{E}+01$ & $0.5313724 \mathrm{E}+05$ \\
16 & $0.1087129 \mathrm{E}-03$ & $0.6113238 \mathrm{E}+01$ & $0.5623286 \mathrm{E}+05$ \\
18 & $0.1073385 \mathrm{E}-03$ & $0.6286398 \mathrm{E}+01$ & $0.5856611 \mathrm{E}+05$ \\
20 & $0.1063557 \mathrm{E}-03$ & $0.6420884 \mathrm{E}+01$ & $0.6037182 \mathrm{E}+05$ \\
22 & $0.1056283 \mathrm{E}-03$ & $0.6527970 \mathrm{E}+01$ & $0.6180136 \mathrm{E}+05$ \\
24 & $0.1050747 \mathrm{E}-03$ & $0.6615018 \mathrm{E}+01$ & $0.6295541 \mathrm{E}+05$ \\
26 & $0.1046435 \mathrm{E}-03$ & $0.6687020 \mathrm{E}+01$ & $0.6390288 \mathrm{E}+05$ \\
28 & $0.1043011 \mathrm{E}-03$ & $0.6747467 \mathrm{E}+01$ & $0.6469222 \mathrm{E}+05$ \\
30 & $0.1040246 \mathrm{E}-03$ & $0.6798866 \mathrm{E}+01$ & $0.6535827 \mathrm{E}+05$ \\
32 & $0.1037981 \mathrm{E}-03$ & $0.6843058 \mathrm{E}+01$ & $0.6592663 \mathrm{E}+05$ \\
34 & $0.1036102 \mathrm{E}-03$ & $0.6881427 \mathrm{E}+01$ & $0.6641649 \mathrm{E}+05$ \\
36 & $0.1034527 \mathrm{E}-03$ & $0.6915028 \mathrm{E}+01$ & $0.6684243 \mathrm{E}+05$ \\
38 & $0.1033192 \mathrm{E}-03$ & $0.6944680 \mathrm{E}+01$ & $0.6721575 \mathrm{E}+05$ \\
40 & $0.1032052 \mathrm{E}-03$ & $0.6971026 \mathrm{E}+01$ & $0.6754528 \mathrm{E}+05$ \\
50 & $0.1028247 \mathrm{E}-03$ & $0.7068096 \mathrm{E}+01$ & $0.6873930 \mathrm{E}+05$
\end{tabular}


Table 9.13

Extreme $\beta_{N, N}$-singular values $w /$ condition nos: $L_{N, N}, a_{0}=-34.05, a_{1}=6$

\begin{tabular}{|r|c|c|c|}
\hline \multicolumn{1}{|c|}{$N$} & min. $\beta$-sing. & max. $\beta$-sing. & condition no. \\
\hline 8 & $0.1278167 \mathrm{E}-03$ & $0.4386774 \mathrm{E}+01$ & $0.3432082 \mathrm{E}+05$ \\
10 & $0.1198081 \mathrm{E}-03$ & $0.5093922 \mathrm{E}+01$ & $0.4251733 \mathrm{E}+05$ \\
12 & $0.1146689 \mathrm{E}-03$ & $0.5545806 \mathrm{E}+01$ & $0.4836364 \mathrm{E}+05$ \\
14 & $0.1115933 \mathrm{E}-03$ & $0.5859062 \mathrm{E}+01$ & $0.5250372 \mathrm{E}+05$ \\
16 & $0.1096036 \mathrm{E}-03$ & $0.6088021 \mathrm{E}+01$ & $0.5554582 \mathrm{E}+05$ \\
18 & $0.1082408 \mathrm{E}-03$ & $0.6261710 \mathrm{E}+01$ & $0.5784981 \mathrm{E}+05$ \\
20 & $0.1072658 \mathrm{E}-03$ & $0.6397332 \mathrm{E}+01$ & $0.5964000 \mathrm{E}+05$ \\
22 & $0.1065438 \mathrm{E}-03$ & $0.6505755 \mathrm{E}+01$ & $0.6106176 \mathrm{E}+05$ \\
24 & $0.1059942 \mathrm{E}-03$ & $0.6594157 \mathrm{E}+01$ & $0.6221245 \mathrm{E}+05$ \\
26 & $0.1055658 \mathrm{E}-03$ & $0.6667451 \mathrm{E}+01$ & $0.6315918 \mathrm{E}+05$ \\
28 & $0.1052255 \mathrm{E}-03$ & $0.6729095 \mathrm{E}+01$ & $0.6394925 \mathrm{E}+05$ \\
30 & $0.1049505 \mathrm{E}-03$ & $0.6781588 \mathrm{E}+01$ & $0.6461699 \mathrm{E}+05$ \\
32 & $0.1047254 \mathrm{E}-03$ & $0.6826776 \mathrm{E}+01$ & $0.6518743 \mathrm{E}+05$ \\
34 & $0.1045383 \mathrm{E}-03$ & $0.6866049 \mathrm{E}+01$ & $0.6567977 \mathrm{E}+05$ \\
36 & $0.1043816 \mathrm{E}-03$ & $0.6900471 \mathrm{E}+01$ & $0.6610808 \mathrm{E}+05$ \\
38 & $0.1042485 \mathrm{E}-03$ & $0.6930868 \mathrm{E}+01$ & $0.6648412 \mathrm{E}+05$ \\
40 & $0.1041352 \mathrm{E}-03$ & $0.6957894 \mathrm{E}+01$ & $0.6681596 \mathrm{E}+05$ \\
50 & $0.1037555 \mathrm{E}-03$ & $0.7057599 \mathrm{E}+01$ & $0.6802144 \mathrm{E}+05$ \\
\hline
\end{tabular}

Table 9.14

Extreme Eigenvalues w/ spectral condition nos: $L_{N, N}, a_{0}=-22.1725, a_{1}=3.5$

\begin{tabular}{|r|c|c|c|}
\hline$N$ & min. eig. & max. eig. & condition no. \\
\hline 8 & $0.1234644 \mathrm{E}+00$ & $0.3862221 \mathrm{E}+01$ & $0.3128205 \mathrm{E}+02$ \\
10 & $0.1158532 \mathrm{E}+00$ & $0.4573414 \mathrm{E}+01$ & $0.3947592 \mathrm{E}+02$ \\
12 & $0.1122258 \mathrm{E}+00$ & $0.5040091 \mathrm{E}+01$ & $0.4491026 \mathrm{E}+02$ \\
14 & $0.1102098 \mathrm{E}+00$ & $0.5374463 \mathrm{E}+01$ & $0.4876575 \mathrm{E}+02$ \\
16 & $0.1089700 \mathrm{E}+00$ & $0.5626984 \mathrm{E}+01$ & $0.5163792 \mathrm{E}+02$ \\
18 & $0.1081510 \mathrm{E}+00$ & $0.5824507 \mathrm{E}+01$ & $0.5385533 \mathrm{E}+02$ \\
20 & $0.1075804 \mathrm{E}+00$ & $0.5983128 \mathrm{E}+01$ & $0.5561539 \mathrm{E}+02$ \\
22 & $0.1071663 \mathrm{E}+00$ & $0.6113208 \mathrm{E}+01$ & $0.5704411 \mathrm{E}+02$ \\
24 & $0.1068559 \mathrm{E}+00$ & $0.6221745 \mathrm{E}+01$ & $0.5822557 \mathrm{E}+02$ \\
26 & $0.1066169 \mathrm{E}+00$ & $0.6313633 \mathrm{E}+01$ & $0.5921794 \mathrm{E}+02$ \\
28 & $0.1064289 \mathrm{E}+00$ & $0.6392398 \mathrm{E}+01$ & $0.6006264 \mathrm{E}+02$ \\
30 & $0.1062782 \mathrm{E}+00$ & $0.6460643 \mathrm{E}+01$ & $0.6078993 \mathrm{E}+02$ \\
32 & $0.1061555 \mathrm{E}+00$ & $0.6520327 \mathrm{E}+01$ & $0.6142243 \mathrm{E}+02$ \\
34 & $0.1060542 \mathrm{E}+00$ & $0.6572957 \mathrm{E}+01$ & $0.6197732 \mathrm{E}+02$ \\
36 & $0.1059697 \mathrm{E}+00$ & $0.6619704 \mathrm{E}+01$ & $0.6246791 \mathrm{E}+02$ \\
38 & $0.1058983 \mathrm{E}+00$ & $0.6661497 \mathrm{E}+01$ & $0.6290467 \mathrm{E}+02$ \\
40 & $0.1058375 \mathrm{E}+00$ & $0.6699080 \mathrm{E}+01$ & $0.6329591 \mathrm{E}+02$ \\
50 & $0.1056357 \mathrm{E}+00$ & $0.6841562 \mathrm{E}+01$ & $0.6476561 \mathrm{E}+02$
\end{tabular}


Table 9.15

Extreme singular values $w /$ condition nos: $L_{N, N}, a_{0}=-22.1725, a_{1}=3.5$

\begin{tabular}{|r|c|c|c|}
\hline \multicolumn{1}{|c|}{$N$} & min. sing. & max. sing. & condition no. \\
\hline 8 & $0.4261767 \mathrm{E}-02$ & $0.4770060 \mathrm{E}+01$ & $0.1119268 \mathrm{E}+04$ \\
10 & $0.3974212 \mathrm{E}-02$ & $0.5380431 \mathrm{E}+01$ & $0.1353836 \mathrm{E}+04$ \\
12 & $0.3818710 \mathrm{E}-02$ & $0.5769969 \mathrm{E}+01$ & $0.1510973 \mathrm{E}+04$ \\
14 & $0.3724948 \mathrm{E}-02$ & $0.6039779 \mathrm{E}+01$ & $0.1621440 \mathrm{E}+04$ \\
16 & $0.3663956 \mathrm{E}-02$ & $0.6236708 \mathrm{E}+01$ & $0.1702179 \mathrm{E}+04$ \\
18 & $0.3622016 \mathrm{E}-02$ & $0.6386079 \mathrm{E}+01$ & $0.1763128 \mathrm{E}+04$ \\
20 & $0.3591925 \mathrm{E}-02$ & $0.6502862 \mathrm{E}+01$ & $0.1810411 \mathrm{E}+04$ \\
22 & $0.3569595 \mathrm{E}-02$ & $0.6596437 \mathrm{E}+01$ & $0.1847951 \mathrm{E}+04$ \\
24 & $0.3552565 \mathrm{E}-02$ & $0.6672956 \mathrm{E}+01$ & $0.1878349 \mathrm{E}+04$ \\
26 & $0.3539279 \mathrm{E}-02$ & $0.6736602 \mathrm{E}+01$ & $0.1903383 \mathrm{E}+04$ \\
28 & $0.3528712 \mathrm{E}-02$ & $0.6790313 \mathrm{E}+01$ & $0.1924303 \mathrm{E}+04$ \\
30 & $0.3520170 \mathrm{E}-02$ & $0.6836207 \mathrm{E}+01$ & $0.1942011 \mathrm{E}+04$ \\
32 & $0.3513165 \mathrm{E}-02$ & $0.6875847 \mathrm{E}+01$ & $0.1957166 \mathrm{E}+04$ \\
34 & $0.3507350 \mathrm{E}-02$ & $0.6910412 \mathrm{E}+01$ & $0.1970266 \mathrm{E}+04$ \\
36 & $0.3502469 \mathrm{E}-02$ & $0.6940802 \mathrm{E}+01$ & $0.1981688 \mathrm{E}+04$ \\
38 & $0.3498333 \mathrm{E}-02$ & $0.6967722 \mathrm{E}+01$ & $0.1991727 \mathrm{E}+04$ \\
40 & $0.3494796 \mathrm{E}-02$ & $0.6991726 \mathrm{E}+01$ & $0.2000610 \mathrm{E}+04$ \\
50 & $0.3482976 \mathrm{E}-02$ & $0.7080935 \mathrm{E}+01$ & $0.2033013 \mathrm{E}+04$ \\
\hline
\end{tabular}

Table 9.16

Extreme $\beta_{N, N}$-singular values $w /$ condition nos: $L_{N, N}, a_{0}=-22.1725, a_{1}=3.5$

\begin{tabular}{|r|c|c|c|}
\hline$N$ & min. $\beta$-sing. & max. $\beta$-sing. & condition no. \\
\hline 8 & $0.4276759 \mathrm{E}-02$ & $0.4766816 \mathrm{E}+01$ & $0.1114586 \mathrm{E}+04$ \\
10 & $0.3992227 \mathrm{E}-02$ & $0.5373119 \mathrm{E}+01$ & $0.1345895 \mathrm{E}+04$ \\
12 & $0.3839581 \mathrm{E}-02$ & $0.5759006 \mathrm{E}+01$ & $0.1499905 \mathrm{E}+04$ \\
14 & $0.3748074 \mathrm{E}-02$ & $0.6026895 \mathrm{E}+01$ & $0.1607998 \mathrm{E}+04$ \\
16 & $0.3688794 \mathrm{E}-02$ & $0.6223216 \mathrm{E}+01$ & $0.1687060 \mathrm{E}+04$ \\
18 & $0.3648152 \mathrm{E}-02$ & $0.6372701 \mathrm{E}+01$ & $0.1746830 \mathrm{E}+04$ \\
20 & $0.3619053 \mathrm{E}-02$ & $0.6489940 \mathrm{E}+01$ & $0.1793270 \mathrm{E}+04$ \\
22 & $0.3597493 \mathrm{E}-02$ & $0.6584113 \mathrm{E}+01$ & $0.1830195 \mathrm{E}+04$ \\
24 & $0.3581070 \mathrm{E}-02$ & $0.6661269 \mathrm{E}+01$ & $0.1860134 \mathrm{E}+04$ \\
26 & $0.3568268 \mathrm{E}-02$ & $0.6725543 \mathrm{E}+01$ & $0.1884820 \mathrm{E}+04$ \\
28 & $0.3558094 \mathrm{E}-02$ & $0.6779851 \mathrm{E}+01$ & $0.1905473 \mathrm{E}+04$ \\
30 & $0.3549873 \mathrm{E}-02$ & $0.6826302 \mathrm{E}+01$ & $0.1922970 \mathrm{E}+04$ \\
32 & $0.3543136 \mathrm{E}-02$ & $0.6866457 \mathrm{E}+01$ & $0.1937961 \mathrm{E}+04$ \\
34 & $0.3537544 \mathrm{E}-02$ & $0.6901495 \mathrm{E}+01$ & $0.1950929 \mathrm{E}+04$ \\
36 & $0.3532852 \mathrm{E}-02$ & $0.6932321 \mathrm{E}+01$ & $0.1962245 \mathrm{E}+04$ \\
38 & $0.3528876 \mathrm{E}-02$ & $0.6959640 \mathrm{E}+01$ & $0.1972197 \mathrm{E}+04$ \\
40 & $0.3525478 \mathrm{E}-02$ & $0.6984011 \mathrm{E}+01$ & $0.1981011 \mathrm{E}+04$ \\
50 & $0.3514122 \mathrm{E}-02$ & $0.7074674 \mathrm{E}+01$ & $0.2013212 \mathrm{E}+04$ \\
\hline
\end{tabular}


Additionally, we are concerned with the distribution of the $\beta_{N, N}$-singular values of the preconditioned matrix $L_{N, N}$. In particular, we are interested in the clustering of the

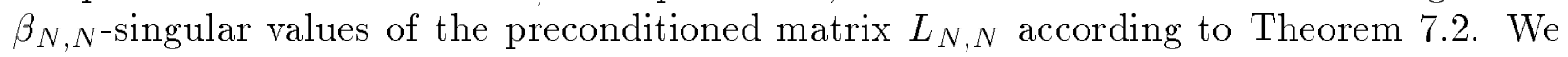
computed the number of $\beta_{N, N^{-}}$-singular values as well as the number of singular values outside the interval given in Theorem 7.2 and report the results in Tables $9.17-9.20$. The columns entitled "lower" and "upper" are the computed lower and upper bounds for the clustering interval given by Theorem 7.2. The values are computed by first computing the eigenvalues of $\tilde{\beta}_{N, M} W_{N, M} \hat{B}_{N, M}$. These numerical results of $\beta_{N, N}$-singular values confirm the results of Theorem 7.2. Although we do not have a theoretical justification for it, the numerical results reported in these tables suggest that the singular values behave similarly to the the $\beta_{N, N^{-}}$singular values.

Table 9.17

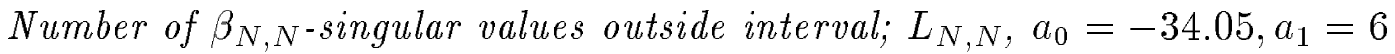

\begin{tabular}{|c|c|c|c|}
\hline$N$ & No. outside & lower & upper \\
\hline 10 & 5 & $0.4282405 \mathrm{E}+00$ & $0.1559045 \mathrm{E}+02$ \\
12 & 5 & $0.4149416 \mathrm{E}+00$ & $0.1614158 \mathrm{E}+02$ \\
14 & 5 & $0.4062323 \mathrm{E}+00$ & $0.1658141 \mathrm{E}+02$ \\
16 & 5 & $0.4001191 \mathrm{E}+00$ & $0.1693874 \mathrm{E}+02$ \\
18 & 5 & $0.3956103 \mathrm{E}+00$ & $0.1723368 \mathrm{E}+02$ \\
20 & 5 & $0.3921580 \mathrm{E}+00$ & $0.1748059 \mathrm{E}+02$ \\
22 & 5 & $0.3894362 \mathrm{E}+00$ & $0.1768995 \mathrm{E}+02$ \\
24 & 6 & $0.3872393 \mathrm{E}+00$ & $0.1786949 \mathrm{E}+02$ \\
26 & 6 & $0.3854311 \mathrm{E}+00$ & $0.1802501 \mathrm{E}+02$ \\
28 & 6 & $0.3839187 \mathrm{E}+00$ & $0.1816095 \mathrm{E}+02$ \\
30 & 6 & $0.3826361 \mathrm{E}+00$ & $0.1828072 \mathrm{E}+02$ \\
32 & 6 & $0.3815353 \mathrm{E}+00$ & $0.1838700 \mathrm{E}+02$ \\
34 & 6 & $0.3805809 \mathrm{E}+00$ & $0.1848192 \mathrm{E}+02$ \\
36 & 6 & $0.3797459 \mathrm{E}+00$ & $0.1856720 \mathrm{E}+02$ \\
38 & 6 & $0.3790094 \mathrm{E}+00$ & $0.1864421 \mathrm{E}+02$ \\
40 & 6 & $0.3783552 \mathrm{E}+00$ & $0.1871409 \mathrm{E}+02$ \\
50 & 6 & $0.3759452 \mathrm{E}+00$ & $0.1898452 \mathrm{E}+02$ \\
\hline
\end{tabular}


Table 9.18

Number of singular values outside interval; $L_{N, N}, a_{0}=-34.05, a_{1}=6$

\begin{tabular}{|c|r|c|c|}
\hline$N$ & No. outside & lower & upper \\
\hline 10 & 5 & $0.4282405 \mathrm{E}+00$ & $0.1559045 \mathrm{E}+02$ \\
12 & 5 & $0.4149416 \mathrm{E}+00$ & $0.1614158 \mathrm{E}+02$ \\
14 & 5 & $0.4062323 \mathrm{E}+00$ & $0.1658141 \mathrm{E}+02$ \\
16 & 5 & $0.4001191 \mathrm{E}+00$ & $0.1693874 \mathrm{E}+02$ \\
18 & 6 & $0.3956103 \mathrm{E}+00$ & $0.1723368 \mathrm{E}+02$ \\
20 & 6 & $0.3921580 \mathrm{E}+00$ & $0.1748059 \mathrm{E}+02$ \\
22 & 6 & $0.3894362 \mathrm{E}+00$ & $0.1768995 \mathrm{E}+02$ \\
24 & 6 & $0.3872393 \mathrm{E}+00$ & $0.1786949 \mathrm{E}+02$ \\
26 & 6 & $0.3854311 \mathrm{E}+00$ & $0.1802501 \mathrm{E}+02$ \\
28 & 6 & $0.3839187 \mathrm{E}+00$ & $0.1816095 \mathrm{E}+02$ \\
30 & 6 & $0.3826361 \mathrm{E}+00$ & $0.1828072 \mathrm{E}+02$ \\
32 & 6 & $0.3815353 \mathrm{E}+00$ & $0.1838700 \mathrm{E}+02$ \\
34 & 6 & $0.3805809 \mathrm{E}+00$ & $0.1848192 \mathrm{E}+02$ \\
36 & 6 & $0.3797459 \mathrm{E}+00$ & $0.1856720 \mathrm{E}+02$ \\
38 & 6 & $0.3790094 \mathrm{E}+00$ & $0.1864421 \mathrm{E}+02$ \\
40 & 6 & $0.3783552 \mathrm{E}+00$ & $0.1871409 \mathrm{E}+02$ \\
50 & 6 & $0.3759452 \mathrm{E}+00$ & $0.1898452 \mathrm{E}+02$ \\
\hline
\end{tabular}

Table 9.19

Number of $\beta_{N, N}$-singular values outside interval; $L_{N, N}, a_{0}=-22.1725, a_{1}=3.5$

\begin{tabular}{|c|r|c|c|}
\hline$N$ & No. outside & lower & upper \\
\hline 10 & 4 & $0.4309650 \mathrm{E}+00$ & $0.1531501 \mathrm{E}+02$ \\
12 & 4 & $0.4167516 \mathrm{E}+00$ & $0.1594577 \mathrm{E}+02$ \\
14 & 4 & $0.4075116 \mathrm{E}+00$ & $0.1643620 \mathrm{E}+02$ \\
16 & 4 & $0.4010664 \mathrm{E}+00$ & $0.1682733 \mathrm{E}+02$ \\
18 & 4 & $0.3963373 \mathrm{E}+00$ & $0.1714577 \mathrm{E}+02$ \\
20 & 4 & $0.3927322 \mathrm{E}+00$ & $0.1740963 \mathrm{E}+02$ \\
22 & 4 & $0.3899004 \mathrm{E}+00$ & $0.1763156 \mathrm{E}+02$ \\
24 & 4 & $0.3876218 \mathrm{E}+00$ & $0.1782067 \mathrm{E}+02$ \\
26 & 4 & $0.3857515 \mathrm{E}+00$ & $0.1798362 \mathrm{E}+02$ \\
28 & 4 & $0.3841908 \mathrm{E}+00$ & $0.1812544 \mathrm{E}+02$ \\
30 & 4 & $0.3828698 \mathrm{E}+00$ & $0.1824993 \mathrm{E}+02$ \\
32 & 4 & $0.3817382 \mathrm{E}+00$ & $0.1836007 \mathrm{E}+02$ \\
34 & 4 & $0.3807586 \mathrm{E}+00$ & $0.1845817 \mathrm{E}+02$ \\
36 & 4 & $0.3799028 \mathrm{E}+00$ & $0.1854610 \mathrm{E}+02$ \\
38 & 4 & $0.3791489 \mathrm{E}+00$ & $0.1862535 \mathrm{E}+02$ \\
40 & 4 & $0.3784801 \mathrm{E}+00$ & $0.1869713 \mathrm{E}+02$ \\
50 & 4 & $0.3760224 \mathrm{E}+00$ & $0.1897385 \mathrm{E}+02$ \\
\hline
\end{tabular}


Table 9.20

Number of singular values outside interval; $L_{N, N}, a_{0}=-22.1725, a_{1}=3.5$

\begin{tabular}{|c|c|c|c|}
\hline$N$ & No. outside & lower & upper \\
\hline 10 & 4 & $0.4309650 \mathrm{E}+00$ & $0.1531501 \mathrm{E}+02$ \\
12 & 4 & $0.4167516 \mathrm{E}+00$ & $0.1594577 \mathrm{E}+02$ \\
14 & 4 & $0.4075116 \mathrm{E}+00$ & $0.1643620 \mathrm{E}+02$ \\
16 & 4 & $0.4010664 \mathrm{E}+00$ & $0.1682733 \mathrm{E}+02$ \\
18 & 5 & $0.3963373 \mathrm{E}+00$ & $0.1714577 \mathrm{E}+02$ \\
20 & 5 & $0.3927322 \mathrm{E}+00$ & $0.1740963 \mathrm{E}+02$ \\
22 & 5 & $0.3899004 \mathrm{E}+00$ & $0.1763156 \mathrm{E}+02$ \\
24 & 5 & $0.3876218 \mathrm{E}+00$ & $0.1782067 \mathrm{E}+02$ \\
26 & 5 & $0.3857515 \mathrm{E}+00$ & $0.1798362 \mathrm{E}+02$ \\
28 & 5 & $0.3841908 \mathrm{E}+00$ & $0.1812544 \mathrm{E}+02$ \\
30 & 5 & $0.3828698 \mathrm{E}+00$ & $0.1824993 \mathrm{E}+02$ \\
32 & 5 & $0.3817382 \mathrm{E}+00$ & $0.1836007 \mathrm{E}+02$ \\
34 & 5 & $0.3807586 \mathrm{E}+00$ & $0.1845817 \mathrm{E}+02$ \\
36 & 5 & $0.3799028 \mathrm{E}+00$ & $0.1854610 \mathrm{E}+02$ \\
38 & 5 & $0.3791489 \mathrm{E}+00$ & $0.1862535 \mathrm{E}+02$ \\
40 & 5 & $0.3784801 \mathrm{E}+00$ & $0.1869713 \mathrm{E}+02$ \\
50 & 5 & $0.3760224 \mathrm{E}+00$ & $0.1897385 \mathrm{E}+02$ \\
\hline
\end{tabular}

The clustering of $\beta$-singular as well as singular values may be seen graphically in Figures 9.1 to 9.4 .

Figure 9.1

$\beta$-Singular Value Distribution of $L_{N, N}, a_{0}=-22.1725, a_{1}=3.5$

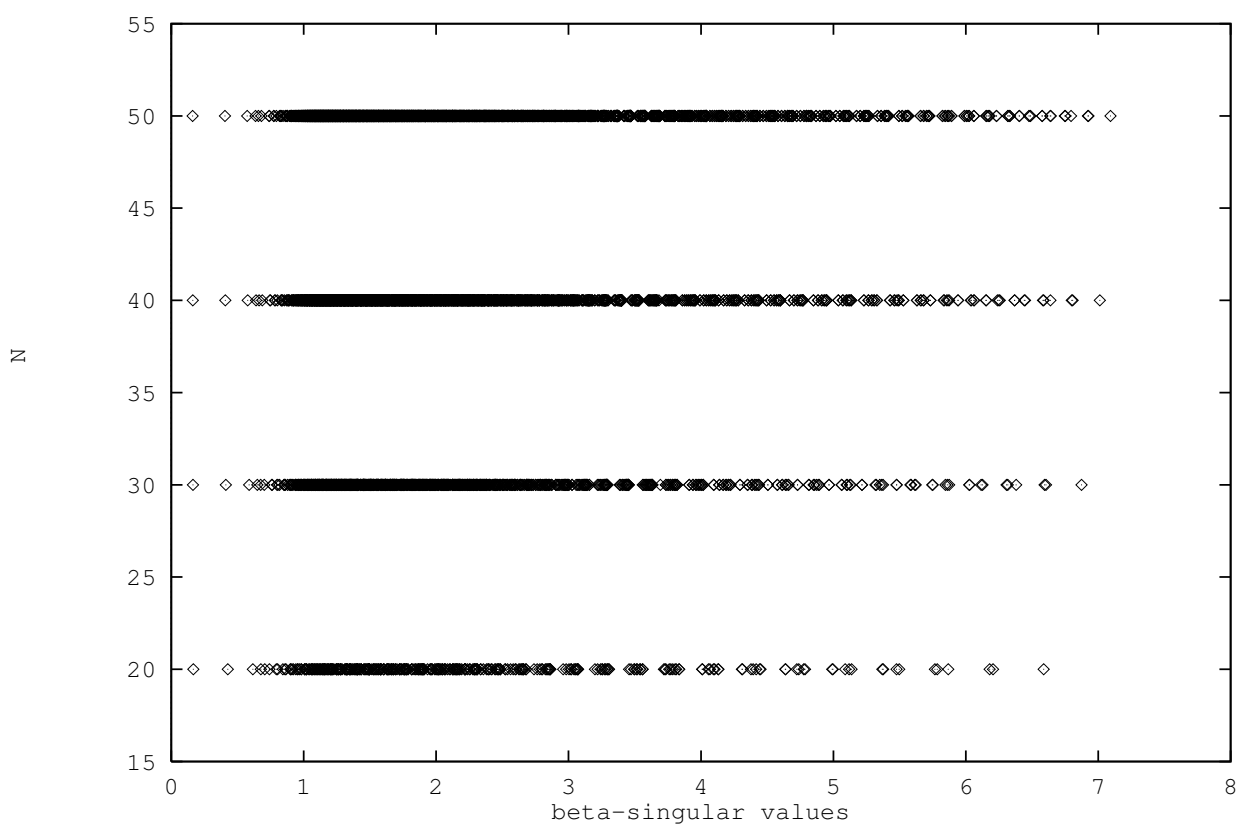


Figure 9.2 Singular Value Distribution of $L_{N, N}, a_{0}=-22.1725, a_{1}=3.5$

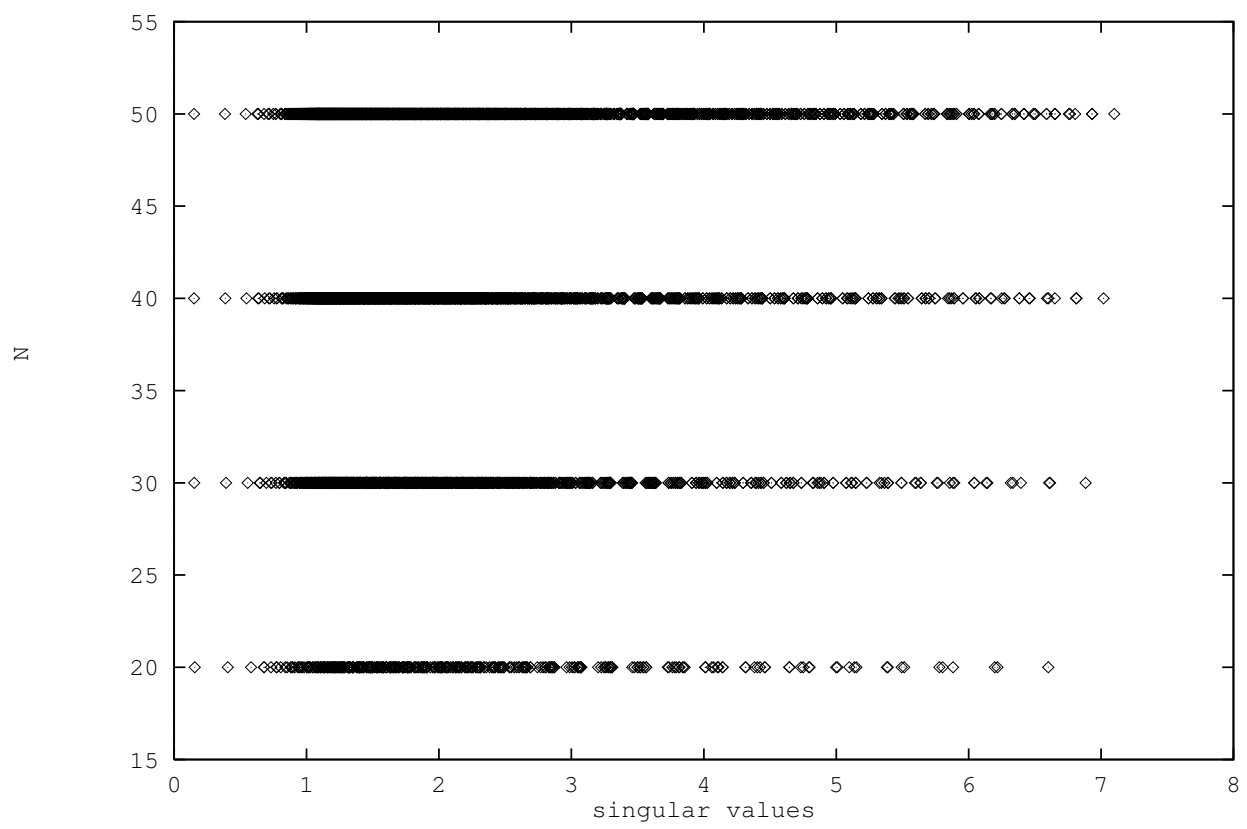

Figure $9.3 \beta$-Singular Value Distribution of $L_{N, N}, a_{0}=-34.05, a_{1}=6$

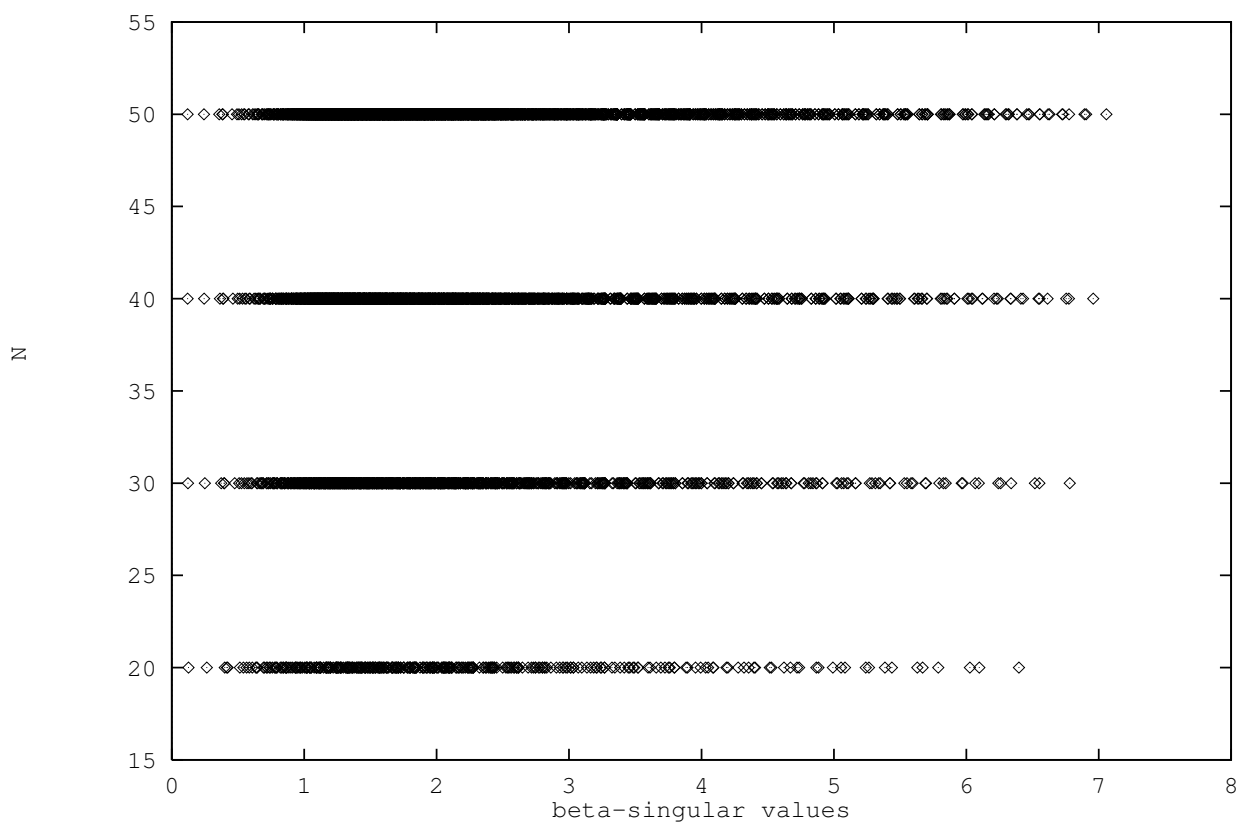


Figure 9.4 Singular Value Distribution of $L_{N, N}, a_{0}=-34.05, a_{1}=6$

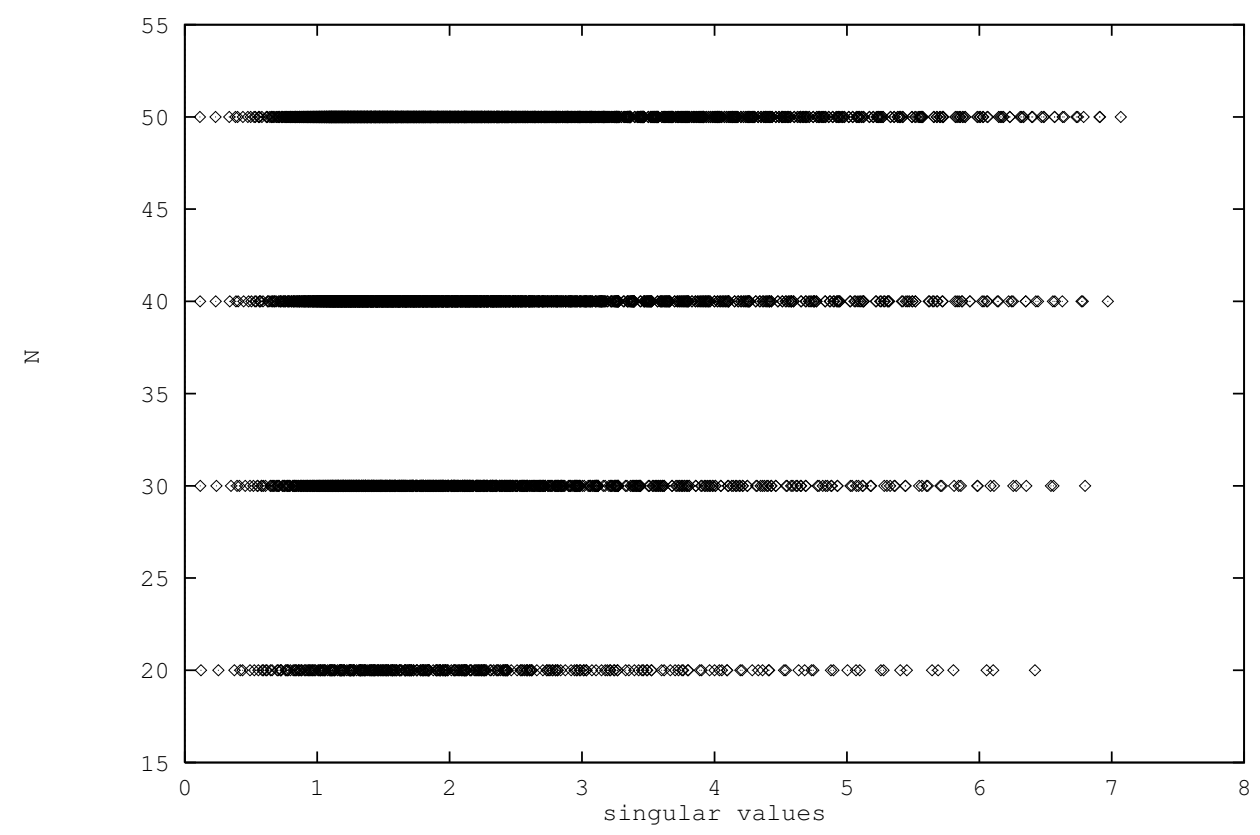

In the case where $a_{0}>0$, the operator $A$ has all positive eigenvalues and so does its pseudospectral approximation $A_{N, N}$ for all values of $N \geq 8$ that we tested. The eigenvalues, singular and $\beta_{N, N}$-singular values of the preconditioned matrix $L_{N, N}$ are exhibited in Tables $9.21-9.23$. These results are representative of problems that we tested having all positive eigenvalues. The eigenvalues, singular and $\beta_{N, N}$-singular values are bounded independent of $N$ and are clustered so that they yield quite small condition numbers. 
Table 9.21

Extreme Eigenvalues w/ spectral condition nos: $L_{N, N}, a_{0}=\left(\frac{5}{4} \pi\right)^{2}, a_{1}=10$.

\begin{tabular}{|c|c|c|c|}
\hline$N$ & min. eig & max. eig & condition no. \\
\hline 8 & $0.1338860 \mathrm{E}+01$ & $0.5668794 \mathrm{E}+01$ & $0.4234045 \mathrm{E}+01$ \\
10 & $0.1342297 \mathrm{E}+01$ & $0.5807957 \mathrm{E}+01$ & $0.4326879 \mathrm{E}+01$ \\
12 & $0.1355080 \mathrm{E}+01$ & $0.5891385 \mathrm{E}+01$ & $0.4347628 \mathrm{E}+01$ \\
14 & $0.1369836 \mathrm{E}+01$ & $0.5960662 \mathrm{E}+01$ & $0.4351368 \mathrm{E}+01$ \\
16 & $0.1383634 \mathrm{E}+01$ & $0.6026450 \mathrm{E}+01$ & $0.4355522 \mathrm{E}+01$ \\
18 & $0.1395368 \mathrm{E}+01$ & $0.6090501 \mathrm{E}+01$ & $0.4364799 \mathrm{E}+01$ \\
20 & $0.1401319 \mathrm{E}+01$ & $0.6152401 \mathrm{E}+01$ & $0.4390434 \mathrm{E}+01$ \\
22 & $0.1373448 \mathrm{E}+01$ & $0.6211488 \mathrm{E}+01$ & $0.4522550 \mathrm{E}+01$ \\
24 & $0.1340351 \mathrm{E}+01$ & $0.6267309 \mathrm{E}+01$ & $0.4675872 \mathrm{E}+01$ \\
26 & $0.1312209 \mathrm{E}+01$ & $0.6319664 \mathrm{E}+01$ & $0.4816051 \mathrm{E}+01$ \\
28 & $0.1288272 \mathrm{E}+01$ & $0.6368538 \mathrm{E}+01$ & $0.4943474 \mathrm{E}+01$ \\
30 & $0.1267689 \mathrm{E}+01$ & $0.6414039 \mathrm{E}+01$ & $0.5059630 \mathrm{E}+01$ \\
32 & $0.1249812 \mathrm{E}+01$ & $0.6456343 \mathrm{E}+01$ & $0.5165852 \mathrm{E}+01$ \\
34 & $0.1234145 \mathrm{E}+01$ & $0.6495663 \mathrm{E}+01$ & $0.5263288 \mathrm{E}+01$ \\
36 & $0.1220307 \mathrm{E}+01$ & $0.6532220 \mathrm{E}+01$ & $0.5352931 \mathrm{E}+01$ \\
38 & $0.1207998 \mathrm{E}+01$ & $0.6566236 \mathrm{E}+01$ & $0.5435636 \mathrm{E}+01$ \\
40 & $0.1196979 \mathrm{E}+01$ & $0.6597924 \mathrm{E}+01$ & $0.5512145 \mathrm{E}+01$ \\
\hline
\end{tabular}

Table 9.22

Extreme singular values $w /$ condition nos: $L_{N, N}, a_{0}=\left(\frac{5}{4} \pi\right)^{2}, a_{1}=10$.

\begin{tabular}{|r|c|c|c|}
\hline$N$ & min. sing. & max. sing. & condition no. \\
\hline 8 & $0.1096162 \mathrm{E}+01$ & $0.6892226 \mathrm{E}+01$ & $0.6287598 \mathrm{E}+01$ \\
10 & $0.1062761 \mathrm{E}+01$ & $0.6899788 \mathrm{E}+01$ & $0.6492321 \mathrm{E}+01$ \\
12 & $0.1037516 \mathrm{E}+01$ & $0.6908768 \mathrm{E}+01$ & $0.6658948 \mathrm{E}+01$ \\
14 & $0.1007030 \mathrm{E}+01$ & $0.6921852 \mathrm{E}+01$ & $0.6873532 \mathrm{E}+01$ \\
16 & $0.9755505 \mathrm{E}+00$ & $0.6938240 \mathrm{E}+01$ & $0.7112128 \mathrm{E}+01$ \\
18 & $0.9505292 \mathrm{E}+00$ & $0.6956526 \mathrm{E}+01$ & $0.7318582 \mathrm{E}+01$ \\
20 & $0.9313886 \mathrm{E}+00$ & $0.6975512 \mathrm{E}+01$ & $0.7489368 \mathrm{E}+01$ \\
22 & $0.9166251 \mathrm{E}+00$ & $0.6994384 \mathrm{E}+01$ & $0.7630583 \mathrm{E}+01$ \\
24 & $0.9050569 \mathrm{E}+00$ & $0.7012650 \mathrm{E}+01$ & $0.7748297 \mathrm{E}+01$ \\
26 & $0.8958449 \mathrm{E}+00$ & $0.7030044 \mathrm{E}+01$ & $0.7847390 \mathrm{E}+01$ \\
28 & $0.8883985 \mathrm{E}+00$ & $0.7046444 \mathrm{E}+01$ & $0.7931625 \mathrm{E}+01$ \\
30 & $0.8822976 \mathrm{E}+00$ & $0.7061816 \mathrm{E}+01$ & $0.8003894 \mathrm{E}+01$ \\
32 & $0.8772383 \mathrm{E}+00$ & $0.7076175 \mathrm{E}+01$ & $0.8066422 \mathrm{E}+01$ \\
34 & $0.8729974 \mathrm{E}+00$ & $0.7089566 \mathrm{E}+01$ & $0.8120947 \mathrm{E}+01$ \\
36 & $0.8694081 \mathrm{E}+00$ & $0.7102047 \mathrm{E}+01$ & $0.8168830 \mathrm{E}+01$ \\
38 & $0.8663437 \mathrm{E}+00$ & $0.7113682 \mathrm{E}+01$ & $0.8211154 \mathrm{E}+01$ \\
40 & $0.8637069 \mathrm{E}+00$ & $0.7124535 \mathrm{E}+01$ & $0.8248788 \mathrm{E}+01$ \\
\hline
\end{tabular}


Table $\mathbf{9 . 2 3}$

Extreme $\beta_{N, N}$-singular values $w /$ condition nos: $L_{N, N}, a_{0}=\left(\frac{5}{4} \pi\right)^{2}, a_{1}=10$.

\begin{tabular}{|c|c|c|c|}
\hline$N$ & min. $\beta$-sing. & max. $\beta$-sing. & condition no. \\
\hline 8 & $0.1106446 \mathrm{E}+01$ & $0.6886236 \mathrm{E}+01$ & $0.6223742 \mathrm{E}+01$ \\
10 & $0.1075918 \mathrm{E}+01$ & $0.6888207 \mathrm{E}+01$ & $0.6402166 \mathrm{E}+01$ \\
12 & $0.1058669 \mathrm{E}+01$ & $0.6889533 \mathrm{E}+01$ & $0.6507733 \mathrm{E}+01$ \\
14 & $0.1047784 \mathrm{E}+01$ & $0.6897600 \mathrm{E}+01$ & $0.6583035 \mathrm{E}+01$ \\
16 & $0.1040359 \mathrm{E}+01$ & $0.6911276 \mathrm{E}+01$ & $0.6643164 \mathrm{E}+01$ \\
18 & $0.1034990 \mathrm{E}+01$ & $0.6928357 \mathrm{E}+01$ & $0.6694127 \mathrm{E}+01$ \\
20 & $0.1030933 \mathrm{E}+01$ & $0.6947070 \mathrm{E}+01$ & $0.6738626 \mathrm{E}+01$ \\
22 & $0.1027758 \mathrm{E}+01$ & $0.6966227 \mathrm{E}+01$ & $0.6778083 \mathrm{E}+01$ \\
24 & $0.1025203 \mathrm{E}+01$ & $0.6985104 \mathrm{E}+01$ & $0.6813383 \mathrm{E}+01$ \\
26 & $0.1023102 \mathrm{E}+01$ & $0.7003288 \mathrm{E}+01$ & $0.6845151 \mathrm{E}+01$ \\
28 & $0.1021340 \mathrm{E}+01$ & $0.7020567 \mathrm{E}+01$ & $0.6873875 \mathrm{E}+01$ \\
30 & $0.1019841 \mathrm{E}+01$ & $0.7036852 \mathrm{E}+01$ & $0.6899950 \mathrm{E}+01$ \\
32 & $0.1018548 \mathrm{E}+01$ & $0.7052124 \mathrm{E}+01$ & $0.6923706 \mathrm{E}+01$ \\
34 & $0.1017420 \mathrm{E}+01$ & $0.7066408 \mathrm{E}+01$ & $0.6945422 \mathrm{E}+01$ \\
36 & $0.1016426 \mathrm{E}+01$ & $0.7079751 \mathrm{E}+01$ & $0.6965338 \mathrm{E}+01$ \\
38 & $0.1015544 \mathrm{E}+01$ & $0.7092210 \mathrm{E}+01$ & $0.6983659 \mathrm{E}+01$ \\
40 & $0.1014754 \mathrm{E}+01$ & $0.7103847 \mathrm{E}+01$ & $0.7000561 \mathrm{E}+01$
\end{tabular}




\section{References}

[A] P.M. Anselone, "Collectively Compact Operator Approximation Theory and Applications to Integral Equations", Prentic Hall, Englewood Cliffs, N..J., (1971).

[BM] C. Bernardi and Y. Maday, "Polynomial Interpolation Results in Sobolev Spaces", Jour. Comp. Appl. Math 43, , (1992).

[BP] J.H. Bramble and J.E. Pasciak; "Preconditioned iterative methods for nonself-adjoint or indefinite elliptic boundary value problems" in Unification of Finite Elements, $\mathrm{H}$. Kardestuncer (ed.) Elsevier, North-Holland, Amsterdam, 167-184, (1984).

[CG] C. Carlenzoli and P. Gervasio; "Effective numerical algorithms for the solution of algebraic systems arising in spectral methods", University of Minnesota Supercomputer Institute Research Report UMSI 91/137 (1991).

[CHQZ] C. Canuto, M.Y. Hussaini, A. Quarteroni, and T.A. Zang; Spectral Methods in Fluid Dynamics, Springer Verlag, New York 1988.

[CQ] C. Canuto and A. Quarteroni; "Approximation results for orthogonal polynomials in Sobolev spaces", Math. Comp. 38, 67-87 (1982).

[DM] M. Deville and C. Mund; "Finite element preconditioning for pseudospectral solutions of elliptic problems"SIAM J. STAT. Comp. 2 311-342 (1990).

[FMP] V. Faber, T.A. Manteuffel, and S.V. Parter; "On the equivalences of operators and the implications to preconditioned iterative methods for elliptic problems", Advances in Applied Mathematics 11, 109-163 (1990).

[G] C.I. Goldstein, "Spectral distribution of preconditioned elliptic operators and convergence estimates for iterative methods" To Appear: Numer. Funct. Anal. and Optimiz, 14, 45-68 (1993).

[GMP] C.I. Goldstein, T.A. Manteuffel and S.V. Parter, "Preconditioning and boundary conditions without $H_{2}$ estimates: $L_{2}$ condition numbers and the distribution of the singular values" To appear SIAM J. Num. Anal.

[J] C. Johnson; Numerical Solution of Partial Differential Equations by the Finite Element Method. Cambridge University Press, New York, (1987).

[M] Y. Maday, "Résultats d'approximation optimaux pour les operateurs d'interpolation polynomials", C.R. Acad. Sci. Paris, 312, serie 1, 705-710 (1991).

[MP] T.A. Manteuffel and S.V. Parter; "Preconditioning and Boundary Conditions", SIAM. J. Numerical Anal. 27, 656-694 (1990). 
[N] P.G. Nevai, Orthogonal Polynomials, Memoirs of the AMS, Amer. Math. Soc., Providence R.I., (1979).

[Or] S.A. Orszag; "Spectral methods for problems in complex geometries", J. Comp. Physics, 37, 70-92 (1980).

[P1] S.V. Parter; "On the eigenvalues of second order elliptic difference operators", SIAM J. Numer. Anal. 19, 518- 530 (1982).

$[\mathrm{PW}] \quad$ S.V. Parter and S-P. Wong; "Preconditioning second-order elliptic operators: Condition numbers and the distribution of the singular values", Journal of Scientific Computation, 6, 129-157, (1991).

[QZ] A. Quarteroni and E. Zampieri; "Finite element preconditioning for Legendre spectral collocation approximations to elliptic equations and systems", SIAM J. Num. Anal.. 29, 917-936, (1992).

[S] G. Szegö; Orthogonal Polynomials, AMS Colloquium Publications, XXII, fourth ed., Amer. Math. Soc., (1955).

[V] H.A. Van der Vorst; "Bi-CGSTAB: A fast smoothly converging variant of Bi-CG for the solution of non-symmetric linear systems." To appear Scient. and Stat. Comp..

[W1] S-P Wong; "Preconditioning nonconforming finite element methods for treating Dirichlet boundary conditions. I", Numer. Math, 62, 391-411 (1992).

[W2] S-P Wong; "Preconditioning nonconforming finite element methods for treating Dirichlet boundary conditions. II", Numer. Math, 62, 413-437 (1992). 